\title{
Sensor fault diagnosis of singular delayed LPV systems with inexact parameters: an uncertain system approach
}

\author{
Amir Hossein Hassanabadia ${ }^{\text {, Masoud Shafiee }}{ }^{b}$ and Vicenc Puig ${ }^{c}$ \\ ${ }^{a}$ Faculty of Electrical, Biomedical and Mechatronics Engineering, Qazvin Branch, Islamic Azad University, Qazvin, Iran; ${ }^{b}$ Department of Electrical \\ Engineering, Amirkabir University of Technology (Tehran Polytechnic), Tehran, Iran; 'A Automatic Control Department, Technical University of \\ Catalonia (UPC), Barcelona, Spain
}

\begin{abstract}
In this paper, sensor fault diagnosis of a singular delayed linear parameter varying (LPV) system is considered. In the considered system, the model matrices are dependent on some parameters which are real-time measurable. The case of inexact parameter measurements is considered which is close to real situations. Fault diagnosis in this system is achieved via fault estimation. For this purpose, an augmented system is created by including sensor faults as additional system states. Then, an unknown input observer (UIO) is designed which estimates both the system states and the faults in the presence of measurement noise, disturbances and uncertainty induced by inexact measured parameters. Error dynamics and the original system constitute an uncertain system due to inconsistencies between real and measured values of the parameters. Then, the robust estimation of the system states and the faults are achieved with $H_{\infty}$ performance and formulated with a set of linear matrix inequalities (LMIs). The designed UIO is also applicable for fault diagnosis of singular delayed LPV systems with unmeasurable scheduling variables. The efficiency of the proposed approach is illustrated with an example.
\end{abstract}

\section{ARTICLE HISTORY}

Received 5 May 2016

Accepted 1 October 2017

\section{KEYWORDS}

Singular delayed LPV system; unknown input observer (UIO); fault diagnosis; inexact parameters; uncertain system approach

\section{Introduction}

In recent years, fault diagnosis has become an essential part of industrial systems. Great effort has been carried out to develop several approaches for fault diagnosis in different systems. Model-based methods which are based on the comparison between the estimated behaviour of the system using a mathematical model and the physical one obtained from sensor measurements have attracted much attention in the control system community (see Chen and Patton (2012), Ding (2008) and references therein). Fault diagnosis via fault estimation is one of the trends in this area which can be addressed using the descriptor system approach. In this approach, the fault vector is augmented with the state vector that leads to a descriptor system representation for the augmented system. Then, the new state vector can be estimated in the presence of unknown inputs including disturbances and faults (Aouaouda, Chadli, Cocquempot, \& Tarek Khadir, 2013; Gao \& Ding, 2007; López-Estrada, Ponsart, Astorga-Zaragoza, Camas-Anzueto, \& Theilliol, 2015). In this way, the three stages of fault diagnosis, namely fault detection, isolation and estimation, are fulfilled directly in a one-step procedure. Moreover, the residual generation and evaluation are not needed which reduces the computational burden of this method.
Nonlinear systems' fault diagnosis has attracted much attention during the past decades (Bokor \& Szabó, 2009; De Persis \& Isidori, 2001; Frank, 1994; Jiang \& Chowdhury, 2005). One approach to deal with nonlinear systems is the multiple model representation. The local models in this approach can be either linear (Hamdi, Rodrigues, Mechmeche, \& Braiek, 2012a) or nonlinear (Moodi \& Farrokhi, 2013). Linear parameter varying (LPV) systems, also called linear parameter dependent (LPD) systems (Botmart \& Niamsup, 2010; De Souza, Trofino, \& De Oliveira, 2003; Karimi, 2006), are a powerful multiple model representation for nonlinear systems. An LPV system is defined with a linear structure but with parameter varying (dependent) matrices as coefficients of the model. LPV systems were first proposed by Shamma (1988) as a generalisation of the gain-scheduling control systems. The LPV representation has been used for many real applications in various control problems (de Oca, Puig, Witczak, \& Dziekan, 2012; Giarré, Bauso, Falugi, \& Bamieh, 2006; Masubuchi, Kato, Saeki, \& Ohara, 2004; Rodrigues, Sahnoun, Theilliol, \& Ponsart, 2013). The considered parameters in the LPV systems allow representing the entire system behaviour when working in different operating points. These parameters are usually either functions of the system states or functions of the system inputs/outputs. The

CONTACT Amir Hossein Hassanabadi a.hassanabadi@aut.ac.ir

○ 2017 Informa UK Limited, trading as Taylor \& Francis Group 
knowledge of these parameters is needed for adapting the system parameters to the system operating point. In the case that these parameters are functions of the system states, they are unmeasurable and their exact values are not known. In the case that they are functions of the system inputs/outputs, they may be measurable but measurement noises or sensor/actuator faults can deviate them from their true values. So, in both cases the exact knowledge of these parameters may be unavailable or inaccurate. In the observer design for fault diagnosis of LPV systems, the knowledge of these parameters is needed to schedule the observer and as a consequence some uncertainty is induced because of inexact knowledge of parameters. Theilliol and Aberkane (2011) and López-Estrada, Ponsart, Astorga Zaragoza, Theilliol, and Aberkane (2014), Yoneyama (2009) have considered LPV systems with unmeasurable parameters and Jetto and Orsini (2010) have considered these systems with measurable but uncertain parameters. The difference between these two situations is that in the case of an unmeasurable set of parameters, the convergence of parameter estimation error to zero can lead to asymptotic convergence of the observer; but in the case of uncertain or noisy measurable parameters, there is always a bounded error in the state estimation of the observer according to the level of uncertainty or noise in the parameter measurements.

Recently, many researchers have focused on fault diagnosis of singular systems. Fault diagnosis of this type of systems has been considered with a UIO based on eigenstructure assignment in the linear case (Duan, Howe, \& Patton, 2002) and with a fault estimation approach in the nonlinear case (Gao \& Ding, 2007). Singular LPV systems' fault diagnosis has attracted the attention of researchers very recently. Actuator fault estimation for discrete singular LPV systems is carried out in Astorga-Zaragoza, Theilliol, Ponsart, and Rodrigues (2012) and Wang, Rodrigues, Theilliol, and Shen (2015), with the assumption that the exact knowledge of scheduling parameters is available. In Hamdi, Rodrigues, Mechmeche, Theilliol, and Braiek (2012b) and Rodrigues, Hamdi, Braiek, and Theilliol (2014), a proportional integral unknown input observer (PIUIO) for actuator fault detection and isolation (FDI) and a fault tolerant control (FTC) system based on an adaptive observer for continuous singular LPV systems have been designed respectively, both with the assumption that the parameters are exactly known for the observer operation. For the case of unmeasurable parameters, an LPV observer for sensor fault estimation is suggested in López-Estrada et al. (2015) while a robust fault detection observer based on the $H_{-} / H_{\infty}$ approach to characterise the unknown input robustness and the fault sensitivity conditions simultaneously is proposed in Estrada, Ponsart, Theilliol, and Astorga-Zaragoza (2015).

Time delay occurs in the dynamics of many systems which can lead to poor performance or instability. Fault diagnosis in systems which have delayed dynamics has attracted much attention recently. A fault detection filter for both retarded and neutral time delay systems is designed based on the geometric approach in Meskin and Khorasani (2009b) while in Meskin and Khorasani (2009a), FDI for distributed time delay systems has been considered. The problem of robust fault detection based on UIO design for uncertain time delay systems is addressed in Ahmadizadeh, Zarei, and Karimi (2014) such that the fault sensitivity is guaranteed by modelmatching the residual dynamics with a suitable reference model. Fault detection for singular delayed systems has been considered in Chen, Zhong, and Zhang (2011) based on the $H_{\infty}$ fault detection filter and in Zhai, Zhang, and $\mathrm{Li}$ (2014) based on the $H_{-} / H_{\infty}$ approach.

Singular delayed LPV systems have been considered recently. These systems present a general class of nonlinear systems in the LPV format in which delayed dynamics and static relations between states are also considered. The applications of these systems have been considered in open flow canal systems (Hassanabadi, Shafiee, \& Puig, 2016b) and in sewer systems (Hassanabadi, Shafiee, \& Puig, 2016a). Robust admissibility and $H_{\infty}$ filtering of continuous-time systems have been considered in Li and Zhang (2012) and Li and Zhang (2013), respectively, while the robust stability of discretetime counterparts are addressed in Zhang and Zhu (2012). Actuator FDI system design based on perfect unknown input decoupling has been carried out in Hassanabadi et al. (2016b). Hassanabadi et al. (2016a) have considered robust actuator fault detection for these systems based on the $H_{\infty}$ theory of delayed LPV systems and model matching the residual with some suitable reference models to guarantee the minimum fault sensitivity. Systems with singular delayed LPV models such as openflow water networks can be prone to faults in their sensors (flow transmitters). To the best of authors' knowledge, the problem of sensor fault diagnosis in singular delayed LPV systems has not been considered yet.

The goal of this paper is to consider the sensor fault diagnosis problem of singular delayed LPV systems. Moreover, the uncertainty in the scheduling parameters of the system (which are either due to the unmeasurability of the parameters or inexactness in the parameter measurements) will be considered. Singular delayed LPV systems under considering this uncertainty have not been considered yet in the literature. Fault diagnosis is achieved with a direct fault estimation method by making use of the descriptor system approach. In this 
method, the state vector and the sensor fault vector are augmented and then the new state is estimated with a UIO. The advantage of this direct fault diagnosis method is the reduction in computation burden of the diagnosis unit because the residual computation and evaluation steps are not required. In the proposed method, the error dynamics of the estimation procedure depends on both real and inexact parameters. The uncertainty induced by inexact parameters is considered by formulating the error dynamics in an uncertain system structure as in Theilliol and Aberkane (2011) and López-Estrada et al. (2014), Yoneyama (2009). Then, the robust convergence of the designed UIO in terms of the robust stability of the uncertain error dynamics system is addressed with a related bounded real lemma (BRL) and formulated with a set of LMIs. The designed UIO provides the state and fault estimates which can be used for both diagnosis and control.

The remaining of the paper is organised as follows: In Section 2, problem formulation is introduced. In Section 3 , the structure of UIO for the systems under consideration is proposed. In Section 4, the UIO design procedure and fault diagnosis are addressed. In Section 5, the effectiveness of the proposed approach is illustrated with an example. Section 6 concludes the main paper results.

\section{Notation}

Throughout this paper, the following notation will be used. $R$ is the set of real numbers. $I_{n}$ is the $n$-dimensional identity matrix. For a matrix $X, X^{T}$ indicates its transpose. $X^{-1}$ is the inverse and $X^{+}$is the pseudo inverse of $X . *$ is used to show the elements induced by symmetry in a symmetric matrix. $\operatorname{sym}\{A\}$ is a short notation for $A+A^{T}$. For a symmetric matrix $X, X>0(X<0)$ denotes that it is positive (negative) definite. $A \otimes B$ indicates the Kronecker product between the matrices $A$ and $B$. For a square integrable function $x(t)$, its $L_{2}$-norm is defined as $\|x(t)\|_{2}=\sqrt{\int_{0}^{\infty} x(t)^{T} x(t) d t}$.

\section{Problem formulation}

In this paper, a class of singular delayed LPV systems with sensor faults, disturbances and measurement noise is considered:

$$
\left\{\begin{array}{l}
E \dot{x}(t)=A_{0}(\theta(t)) x(t)+A_{1}(\theta(t)) x(t-\tau(t)) \\
\quad+B(\theta(t)) u(t)+R(\theta(t)) d(t) \\
y(t)=C x(t)+D_{f} f(t)+D_{n} n(t) \\
0 \leq \tau(t) \leq \tau_{m} \\
\dot{\tau}(t) \leq \mu \\
x(t)=\phi(t) \quad-\tau_{m}<t<0
\end{array}\right.
$$

where $x(t) \in R^{n_{0}}, u(t) \in R^{k_{u}}, y(t) \in R^{m}, d(t) \in R^{k_{d}}$, $n(t) \in R^{k_{n}}$ and $f(t) \in R^{k_{f}}$ are vectors of states, input signals, output signals, exogenous disturbances, measurement noise and sensor faults, respectively. In (1), $E \in$ $R^{n_{0} \times n_{0}}$ is a constant square matrix that may have rank deficiency $\left(\operatorname{rank}(E)=r \leq n_{0}\right) . A_{0}(\theta(t)), A_{1}(\theta(t)), B(\theta(t))$ and $R(\theta(t))$ are matrices with appropriate dimensions which depend affinely on the time-varying parameter vector $\theta(t) \in R^{l}$ that is real-time measurable. $C, D_{f}$ and $D_{n}$ are constant matrices with appropriate dimensions. $\tau(t)$ is a time-varying delay. $\tau_{m}$ and $\mu$ are the maximum bounds on delay and delay derivative values, respectively. $\phi(t)$ is a continuous vector-valued initial function. The time-varying parameter vector is assumed to be bounded in a hyperbox:

$$
\theta_{k}^{m} \leq \theta_{k}(t) \leq \theta_{k}^{M} \quad k=1, \ldots, l
$$

Definition 2.1 (Dai, 1989): The matrix pencil $(E, A)$ is regular if $\operatorname{det}(s E-A)$ is not identically zero.

Definition 2.2 (Dai, 1989): The matrix pencil $(E, A)$ is impulse-free if $\operatorname{deg}(\operatorname{det}(s E-A))=\operatorname{rank}(E)$.

Definition 2.3 (Li \& Zhang, 2012): System (1) is regular and impulse-free if the matrix pencils $\left(E, A_{0}(\theta(t))\right)$ and $\left(E, A_{0}(\theta(t))+A_{1}(\theta(t))\right)$ are regular and impulsefree for the all domains of $\theta(t)$ defined in (2).

Definition 2.4 (Li \& Zhang, 2012): System (1) is admissible if it is regular, impulse free and stable.

Assumption 2.1: System (1) is assumed to be admissible.

Remark 2.1: For simplicity of notation, the case of linear measurement equation is considered in (1). The methodology presented in this paper could be extended to the case with parameter varying output equation. To see the needed modifications, the interested reader is referred to Ichalal, Marx, Ragot, and Maquin (2009).

The singular delayed LPV system (1) has matrices that depend on the time-varying parameter $\theta(t)$. A common way of treating these systems is to convert them to polytopic format (Hassanabadi et al., 2016b) in which the whole system is represented by a weighted summation of linear subsystems in the $h=2^{l}$ vertices of hyperbox (2). This approach allows extending the methods present in the literature for LTI systems to LPV systems. The associated weights of vertex subsystems are represented by $\rho(\theta(t)) \in R^{h}$ which satisfy:

$$
\begin{aligned}
& 0 \leq \rho_{i}(\theta(t)) \leq 1 \quad i=1, \cdots, h \\
& \sum_{i=1}^{h} \rho_{i}(\theta(t))=1
\end{aligned}
$$


The polytopic representation of (1) is:

$$
\left\{\begin{aligned}
E \dot{x}(t)= & \sum_{i=1}^{h} \rho_{i}(\theta(t))\left[A_{0 i} x(t)+A_{1 i} x(t-\tau(t))\right. \\
& \left.+B_{i} u(t)+R_{i} d(t)\right] \\
y(t)= & C x(t)+D_{f} f(t)+D_{n} n(t)
\end{aligned}\right.
$$

In (5), for every $i \in[1, h]$, the matrices $A_{0 i}, A_{1 i}, B_{i}, R_{i}$, $C, D_{f}$ and $D_{n}$ constitute the linear subsystem defined in the $i$ th vertex of the hyperbox and the summation mechanism in structure (5) allows approximating the parameter varying model (1).

Remark 2.2: The subsystem matrices $A_{0 i}, A_{1 i}, B_{i}, R_{i}$ and the subsystem weights $\rho_{i}(\theta(t))$ can be calculated with the method presented in Hassanabadi et al. (2016a) for any number of parameters.

In order to estimate the states and sensor faults in system (5) simultaneously, a new state vector is constructed by augmenting the state vector of (5) with the fault vector:

$$
\tilde{x}(t)=\left[\begin{array}{l}
x(t) \\
f(t)
\end{array}\right]
$$

The augmented system is presented by:

$$
\left\{\begin{aligned}
\tilde{E} \dot{\tilde{x}}(t)= & \sum_{i=1}^{h} \rho_{i}(\theta(t))\left[\tilde{A}_{0 i} \tilde{x}(t)+\tilde{A}_{1 i} \tilde{x}(t-\tau(t))\right. \\
& \left.+\tilde{\tilde{B}}_{i} u(t)+\tilde{R}_{i} d(t)+\tilde{F} f(t)\right] \\
y(t)= & \tilde{C} \tilde{x}(t)+D_{n} n(t)
\end{aligned}\right.
$$

where

$$
\begin{aligned}
\tilde{E} & =\left[\begin{array}{ll}
E & 0 \\
0 & 0
\end{array}\right], \tilde{A}_{0 i}=\left[\begin{array}{cc}
A_{0 i} & 0 \\
0 & -I_{k_{f}}
\end{array}\right], \\
\tilde{A}_{1 i} & =\left[\begin{array}{cc}
A_{1 i} & 0 \\
0 & 0
\end{array}\right], \tilde{B}_{i}=\left[\begin{array}{c}
B_{i} \\
0
\end{array}\right], \\
\tilde{R}_{i} & =\left[\begin{array}{c}
R_{i} \\
0
\end{array}\right], \tilde{F}=\left[\begin{array}{c}
0 \\
I_{k_{f}}
\end{array}\right], \tilde{C}=\left[\begin{array}{ll}
C & D_{f}
\end{array}\right]
\end{aligned}
$$

Remark 2.3: The order of augmented system (7), is $n=$ $n_{0}+k_{f}$ and all the matrices are with appropriate dimensions.

\section{UIO formulation}

In order to estimate the states of the augmented system (7) in the presence of unknown inputs, the following UIO is proposed:

$$
\left\{\begin{aligned}
\dot{z}(t)= & \sum_{i=1}^{h} \rho_{i}(\hat{\theta}(t))\left[N_{0 i} z(t)+N_{1 i} z(t-\tau(t))\right. \\
& \left.+L_{0 i} y(t)+L_{1 i} y(t-\tau(t))+G_{i} u(t)\right] \\
\hat{\tilde{x}}(t)= & z(t)+H_{2} y(t) \\
\hat{y}(t)= & \tilde{C} \hat{\tilde{x}}(t) \\
z(t)= & 0-\tau_{m}<t<0
\end{aligned}\right.
$$

where $\hat{\tilde{x}}(t) \in R^{n}, \hat{y}(t) \in R^{m}$ and $z(t) \in R^{n}$ are the vectors of the augmented state estimate, output estimate and observer state, respectively. $N_{0 i}, N_{1 i}, L_{0 i}, L_{1 i}, G_{i}$ and $H_{2}$ are observer matrices with appropriate dimensions to be computed. $\hat{\theta}(t)$ is the inexact measured parameters vector and $\rho_{i}(\hat{\theta}(t))$ for $i=1, \ldots, h$ are the weighting functions scheduling between different UIO subsystems corresponding to the inexact measured set of parameters. The state estimation error of UIO (8) is:

$$
e(t)=\tilde{x}(t)-\hat{\tilde{x}}(t) .
$$

According to (7)-(8), the error becomes:

$$
\begin{aligned}
e(t) & =\tilde{x}(t)-z(t)-H_{2} \tilde{C} \tilde{x}(t)-H_{2} D_{n} n(t) \\
& =\left(I_{n}-H_{2} \tilde{C}\right) \tilde{x}(t)-z(t)-H_{2} D_{n} n(t) .
\end{aligned}
$$

Now, a matrix $H_{1} \in R^{n \times n}$ with the following constraint is considered:

$$
H_{1} \tilde{E}=I_{n}-H_{2} \tilde{C}
$$

Thus:

$$
e(t)=H_{1} \tilde{E} \tilde{x}(t)-z(t)-H_{2} D_{n} n(t) .
$$

So, the error dynamics is described by means of:

$$
\dot{e}(t)=H_{1} \tilde{E} \dot{\tilde{x}}(t)-\dot{z}(t)-H_{2} D_{n} \dot{n}(t) .
$$

Substituting (7) and (8) in (13) results in:

$$
\begin{aligned}
\dot{e}(t)= & \sum_{i=1}^{h} \rho_{i}(\theta(t))\left[H_{1} \tilde{A}_{0 i} \tilde{x}(t)+H_{1} \tilde{A}_{1 i} \tilde{x}(t-\tau(t))\right. \\
& \left.+H_{1} \tilde{B}_{i} u(t)+H_{1} \tilde{R}_{i} d(t)+H_{1} \tilde{F} f(t)\right] \\
& -\sum_{i=1}^{h} \rho_{i}(\hat{\theta}(t))\left[N_{0 i} z(t)+N_{1 i} z(t-\tau(t))\right. \\
& +L_{0 i} \tilde{C} \tilde{x}(t)+L_{0 i} D_{n} n(t)+L_{1 i} \tilde{C} \tilde{x}(t-\tau(t)) \\
& \left.+L_{1 i} D_{n} n(t-\tau(t))+G_{i} u(t)\right]-H_{2} D_{n} \dot{n}(t) .
\end{aligned}
$$


Considering (12):

$$
\begin{aligned}
\dot{e}(t)= & \sum_{i=1}^{h} \rho_{i}(\theta(t))\left[H_{1} \tilde{A}_{0 i} \tilde{x}(t)\right. \\
& +H_{1} \tilde{A}_{1 i} \tilde{x}(t-\tau(t))+H_{1} \tilde{B}_{i} u(t) \\
& \left.+H_{1} \tilde{R}_{i} d(t)+H_{1} \tilde{F} f(t)\right]-\sum_{i=1}^{h} \rho_{i}(\hat{\theta}(t))\left[-N_{0 i} e(t)\right. \\
& +N_{0 i} H_{1} \tilde{E} \tilde{x}(t)-N_{0 i} H_{2} D_{n} n(t)-N_{1 i} e(t-\tau(t)) \\
& +N_{1 i} H_{1} \tilde{E} \tilde{x}(t-\tau(t))-N_{1 i} H_{2} D_{n} n(t-\tau(t)) \\
& +L_{0 i} \tilde{C} \tilde{x}(t)+L_{0 i} D_{n} n(t)+L_{1 i} \tilde{C} \tilde{x}(t-\tau(t)) \\
& \left.+L_{1 i} D_{n} n(t-\tau(t))+G_{i} u(t)\right]-H_{2} D_{n} \dot{n}(t) .
\end{aligned}
$$

the following mathematical manipulation of the terms of $\rho_{i}(\hat{\theta}(t))$ :

$$
\begin{aligned}
\sum_{i=1}^{h} & \rho_{i}(\hat{\theta}(t)) X_{i} \\
= & \sum_{i=1}^{h} \rho_{i}(\theta(t)) X_{i}-\sum_{j=1}^{h}\left[\left(\rho_{j}(\theta(t))-\rho_{j}(\hat{\theta}(t))\right] X_{j}\right. \\
= & \sum_{i=1}^{h} \rho_{i}(\theta(t)) X_{i}-\sum_{i=1}^{h} \rho_{i}(\theta(t)) \sum_{j=1}^{h}\left[\rho_{j}(\theta(t))\right. \\
& \left.-\rho_{j}(\hat{\theta}(t))\right] X_{j}
\end{aligned}
$$

allows to transform (15) into:

$$
\begin{aligned}
& \dot{e}(t) \\
& =\sum_{i=1}^{h} \rho_{i}(\theta(t))\left\{\left(H_{1} \tilde{A}_{0 i}-N_{0 i} H_{1} \tilde{E}-L_{0 i} \tilde{C}\right) \tilde{x}(t)+\left(H_{1} \tilde{A}_{1 i}\right.\right. \\
& \left.\quad-N_{1 i} H_{1} \tilde{E}-L_{1 i} \tilde{C}\right) \tilde{x}(t-\tau(t))+N_{0 i} e(t) \\
& \quad+N_{1 i} e(t-\tau(t))+\left(H_{1} \tilde{B}_{i}-G_{i}\right) u(t)+H_{1} \tilde{R}_{i} d(t) \\
& \quad+H_{1} \tilde{F} f(t)+\left(N_{0 i} H_{2} D_{n}-L_{0 i} D_{n}\right) n(t)+\left(N_{1 i} H_{2} D_{n}\right. \\
& \left.\quad-L_{1 i} D_{n}\right) n(t-\tau(t))+\sum_{j=1}^{h}\left(\rho_{j}(\theta(t))-\rho_{j}(\hat{\theta}(t))\right) \\
& \quad \times\left\{\left(N_{0 j} H_{1} \tilde{E}+L_{0 j} \tilde{C}\right) \tilde{x}(t)-N_{0 j} e(t)+\left(N_{1 j} H_{1} \tilde{E}\right.\right. \\
& \left.\quad+L_{1 j} \tilde{C}\right) \tilde{x}(t-\tau(t))-N_{1 j} e(t-\tau(t))+G_{j} u(t) \\
& \quad-\left(N_{0 j} H_{2} D_{n}-L_{0 j} D_{n}\right) n(t)-\left(N_{1 j} H_{2} D_{n}-L_{1 j} D_{n}\right) \\
& \left.\quad \times n(t-\tau(t))\}-H_{2} D_{n} \dot{n}(t)\right\} .
\end{aligned}
$$

If the following conditions are satisfied:

$$
\begin{aligned}
H_{1} \tilde{E}+H_{2} \tilde{C} & =I_{n} \\
N_{0 i} H_{1} \tilde{E}+L_{0 i} \tilde{C} & =H_{1} \tilde{A}_{0 i} \\
N_{1 i} H_{1} \tilde{E}+L_{1 i} \tilde{C} & =H_{1} \tilde{A}_{1 i} \\
G_{i} & =H_{1} \tilde{B}_{i}
\end{aligned}
$$

$$
\begin{aligned}
H_{1} \tilde{R}_{i} & =0 \\
H_{1} \tilde{F} & =0
\end{aligned}
$$

the error dynamics can be written as:

$$
\begin{aligned}
\dot{e}(t)= & \sum_{i=1}^{h} \rho_{i}(\theta(t))\left\{N_{0 i} e(t)+N_{1 i} e(t-\tau(t))+\left(N_{0 i} H_{2} D_{n}\right.\right. \\
& \left.-L_{0 i} D_{n}\right) n(t)+\left(N_{1 i} H_{2} D_{n}-L_{1 i} D_{n}\right) n(t-\tau(t)) \\
& +\sum_{j=1}^{h}\left(\rho_{j}(\theta(t))-\rho_{j}(\hat{\theta}(t))\right)\left\{H_{1} \tilde{A}_{0 j} \tilde{x}(t)-N_{0 j} e(t)\right. \\
& +H_{1} \tilde{A}_{1 j} \tilde{x}(t-\tau(t))-N_{1 j} e(t-\tau(t))+G_{j} u(t) \\
& -\left(N_{0 j} H_{2} D_{n}-L_{0 j} D_{n}\right) n(t)-\left(N_{1 j} H_{2} D_{n}-L_{1 j} D_{n}\right) \\
& \left.\times n(t-\tau(t))\}-H_{2} D_{n} \dot{n}(t)\right\} .
\end{aligned}
$$

Now, by defining a set of new variables as follows:

$$
K_{s i}=L_{s i}-N_{s i} H_{2}
$$

for $s=0,1$ and $i=1, \ldots, h,(19)-(20)$ are transformed to a unified representation:

$$
N_{s i}=H_{1} \tilde{A}_{s i}-K_{s i} \tilde{C}
$$

Moreover, by using (25), (24) is simplified to:

$$
\begin{aligned}
\dot{e}(t)= & \sum_{i=1}^{h} \rho_{i}(\theta(t))\left\{N_{0 i} e(t)+N_{1 i} e(t-\tau(t))-K_{0 i} D_{n} n(t)\right. \\
& -K_{1 i} D_{n} n(t-\tau(t))+\sum_{j=1}^{h}\left(\rho_{j}(\theta(t))-\rho_{j}(\hat{\theta}(t))\right) \\
& \times\left\{H_{1} \tilde{A}_{0 j} \tilde{x}(t)-N_{0 j} e(t)+H_{1} \tilde{A}_{1 j} \tilde{x}(t-\tau(t))\right. \\
& -N_{1 j} e(t-\tau(t))+G_{j} u(t)+K_{0 j} D_{n} n(t) \\
& \left.\left.+K_{1 j} D_{n} n(t-\tau(t))\right\}-H_{2} D_{n} \dot{n}(t)\right\} .
\end{aligned}
$$

Now, by considering a new state vector as

$$
\xi(t)=\left[\tilde{x}(t)^{T} e(t)^{T}\right]^{T}
$$

and a new input vector as

$$
\bar{u}(t)=\left[u(t)^{T} d(t)^{T} f(t)^{T} n(t)^{T} n(t-\tau(t))^{T} \dot{n}(t)^{T}\right]^{T},
$$

the error dynamics system (27) is augmented with system (7) to form the following uncertain system:

$$
\begin{aligned}
\bar{E} \dot{\xi}(t)= & \sum_{i=1}^{h} \rho_{i}(\theta(t))\left\{\left(\bar{A}_{0 i}+\Delta \bar{A}_{0 i}\right) \xi(t)+\left(\bar{A}_{1 i}\right.\right. \\
& \left.\left.+\Delta \bar{A}_{1 i}\right) \xi(t-\tau(t))+\left(\bar{B}_{i}+\Delta \bar{B}_{i}\right) \bar{u}(t)\right\}
\end{aligned}
$$


where

$$
\begin{aligned}
\bar{E} & =\left[\begin{array}{cc}
\tilde{E} & 0 \\
0 & I
\end{array}\right], \bar{A}_{0 i}=\left[\begin{array}{cc}
\tilde{A}_{0 i} & 0 \\
0 & N_{0 i}
\end{array}\right], \bar{A}_{1 i}=\left[\begin{array}{cc}
\tilde{A}_{1 i} & 0 \\
0 & N_{1 i}
\end{array}\right], \\
\bar{B}_{i} & =\left[\begin{array}{ccccc}
\tilde{B}_{i} & \tilde{R}_{i} & \tilde{F} & 0 & 0 \\
0 & 0 & 0 & -K_{0 i} D_{n}-K_{1 i} D_{n}-H_{2} D_{n}
\end{array}\right]
\end{aligned}
$$

and the uncertain terms are defined by:

$$
\begin{aligned}
\Delta \bar{A}_{0 i} & =M_{A_{0}} \Sigma_{A}(t) N_{A} \\
\Delta \bar{A}_{1 i} & =M_{A_{1}} \Sigma_{A}(t) N_{A} \\
\Delta \bar{B}_{i} & =M_{B} \Sigma_{B}(t) N_{B}
\end{aligned}
$$

where ${ }^{1}$

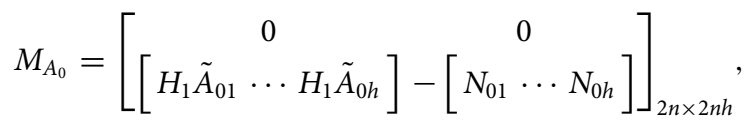

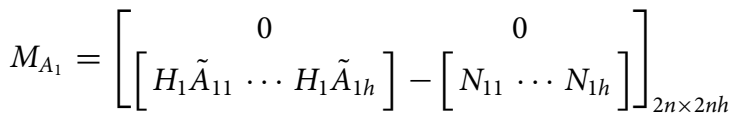

$$
\begin{aligned}
& \Sigma_{A}(t)=\left[\begin{array}{cc}
\Omega_{x}(t) & 0 \\
0 & \Omega_{x}(t)
\end{array}\right]_{2 n h \times 2 n h}, \\
& \Omega_{x}(t)=\operatorname{diag}\left(\delta_{1}(t), \ldots, \delta_{h}(t)\right) \otimes I_{n}, \\
& \delta_{j}(t)=\rho_{j}(\theta(t))-\rho_{j}(\hat{\theta}(t)) \quad(\text { for } j=1, \ldots, h), \\
& N_{A}=\left[\begin{array}{cc}
\phi_{x} & 0 \\
0 & \phi_{x}
\end{array}\right]_{2 n h \times 2 n}, \phi_{x}=\left[\begin{array}{c}
I_{n} \\
\vdots \\
I_{n}
\end{array}\right]_{n h \times n} \\
& M_{B}=
\end{aligned}
$$

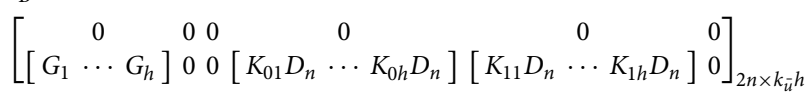

$$
\begin{aligned}
& k_{\bar{u}}=k_{u}+k_{d}+k_{f}+3 k_{n}, \\
& \Sigma_{B}(t)=\operatorname{diag}\left(\Omega_{u}(t), \Omega_{d}, \Omega_{f}, \Omega_{n}(t), \Omega_{n}(t), \bar{\Omega}_{n}\right), \\
& \Omega_{u}(t)=\operatorname{diag}\left(\delta_{1}(t), \ldots, \delta_{h}(t)\right) \otimes I_{k_{u}}, \quad \Omega_{d}=0_{k_{d} h \times k_{d} h}, \\
& \Omega_{f}=0_{k_{f} h \times k_{f} h}, \quad \Omega_{n}(t)=\operatorname{diag}\left(\delta_{1}(t), \ldots, \delta_{h}(t)\right) \otimes I_{k_{n}}, \\
& \bar{\Omega}_{n}=0_{k_{n} h \times k_{n} h} \\
& N_{B}=\operatorname{diag}\left(\phi_{u}, \phi_{d}, \phi_{f}, \phi_{n}, \phi_{n}, \bar{\phi}_{n}\right) \\
& \begin{aligned}
\phi_{u} & =\left[\begin{array}{c}
I_{k_{u}} \\
\vdots \\
I_{k_{u}}
\end{array}\right]_{k_{u} h \times k_{u}}, \quad \phi_{d}=0_{k_{d} h \times k_{d}}, \quad \phi_{f}=0_{k_{f} h \times k_{f}}, \\
\phi_{n} & =\left[\begin{array}{c}
I_{k_{n}} \\
\vdots \\
I_{k_{n}}
\end{array}\right]_{k_{n} h \times k_{n}}, \bar{\phi}_{n}=0_{k_{n} h \times k_{n}}
\end{aligned}
\end{aligned}
$$

Remark 3.1: Due to the convex properties of scheduling functions:

$$
\begin{aligned}
-1 & \leq \underbrace{\rho_{j}(\theta(t))-\rho_{j}(\hat{\theta}(t))}_{\delta_{j}(t)} \leq 1 \\
& \Rightarrow \operatorname{diag}\left(\delta_{1}^{2}(t), \ldots, \delta_{h}^{2}(t)\right) \leq I_{h},
\end{aligned}
$$

So:

$$
\begin{aligned}
& \Sigma_{A}(t)^{T} \Sigma_{A}(t) \leq I_{2 n h} \\
& \Sigma_{B}(t)^{T} \Sigma_{B}(t) \leq I_{k_{\bar{u}} h}
\end{aligned}
$$

\section{UIO design and fault diagnosis}

\subsection{Description and preliminary results}

In this section, the matrices of UIO introduced in (8) are determined such that (18)-(23) are satisfied. By augmenting (18) with (22)-(23), the following matrix equality is obtained:

$$
\underbrace{\left[\begin{array}{ll}
H_{1} & H_{2}
\end{array}\right]}_{\bar{H}} \underbrace{\left[\begin{array}{ccc}
\tilde{E} & \Sigma_{\tilde{R}} & \tilde{F} \\
\tilde{C} & 0 & 0
\end{array}\right]}_{Y}=\underbrace{\left[\begin{array}{lll}
I_{n} & 0 & 0
\end{array}\right]}_{\Psi}
$$

where $\quad \Sigma_{\tilde{R}}=\left[\tilde{R}_{1} \cdots \tilde{R}_{h}\right], \quad \bar{H} \in R^{n \times(n+m)}, \quad Y \in$ $R^{(n+m) \times\left(n+h k_{d}+k_{f}\right)}$ and $\Psi \in R^{n \times\left(n+h k_{d}+k_{f}\right)}$.

Remark 4.1: In the case of a constant disturbance distribution matrix ( $R_{i}=R$ for all subsystems), the formulation derived in (36) is valid by introducing $\Sigma_{\tilde{R}}=\tilde{R}=$ $\left[\begin{array}{ll}R^{T} & 0\end{array}\right]^{T}$ and replacing $h k_{d}$ with $k_{d}$ in the dimension of the related matrices.

Remark 4.2: Matrix equation (36) is solvable if $\operatorname{rank}\left[\begin{array}{l}Y \\ \Psi\end{array}\right]=\operatorname{rank}(Y)$ which is equivalent to the following condition (Koenig, 2005):

$$
\operatorname{rank}(Y)=n+\operatorname{rank}\left(\Sigma_{\tilde{R}}\right)+\operatorname{rank}(\tilde{F})
$$

The solution of matrix equation (36) is:

$$
\bar{H}=\Psi Y^{+}+K\left(I_{n+m}-Y Y^{+}\right)
$$

where $Y^{+}$is the pseudo inverse of matrix $Y$ and $K$ is a gain factor with compatible dimension. This gain adds an additional degree of freedom that helps in the design of a suitable UIO. Then, (38) is partitioned as follows:

$$
\begin{aligned}
\bar{H} & =\left[H_{1} H_{2}\right]=\Psi\left[Y_{1}^{+} Y_{2}^{+}\right]+K\left[V_{1} V_{2}\right] \\
& =[\underbrace{\Psi Y_{1}^{+}}_{H_{10}}+K V_{1} \underbrace{\Psi Y_{2}^{+}}_{H_{20}}+K V_{2}]
\end{aligned}
$$


where $Y_{1}^{+}=Y^{+} T_{1}$ and $Y_{2}^{+}=Y^{+} T_{2}$ in which $T_{1}=$ $\left[\begin{array}{ll}I_{n} & 0_{n \times m}\end{array}\right]^{T}$ and $T_{2}=\left[\begin{array}{ll}0_{m \times n} & I_{m}\end{array}\right]^{T}$. In a similar manner, $V_{1}=V T_{1}$ and $V_{2}=V T_{2}$ in which $V=I_{n+m}-Y Y^{+}$.

Now, a Lemma that will be used to guarantee the robust convergence of UIO is stated:

Lemma 4.1: Consider the following singular delayed LPV system:

$$
\left\{\begin{array}{l}
E \dot{x}(t)=\sum_{i=1}^{h} \rho_{i}(\theta(t))\left[A_{0 i} x(t)+A_{1 i} x(t-\tau(t))+B_{i} w(t)\right] \\
z(t)=C x(t)
\end{array}\right.
$$

where $w(t)$ is a $L_{2}$-norm bounded exogenous input and $z(t)$ is the measured output and all the matrices are with compatible dimensions. For a given $\gamma>0$, if there exist matrices $P$ and $Q>0$ such that the following conditions hold for $i=1, \ldots, h$ :

$$
\begin{gathered}
P^{T} E=E^{T} P \geq 0 \\
\Theta^{i}=\left[\begin{array}{ccc}
P^{T} A_{0 i}+A_{0 i}^{T} P+Q+C^{T} C & P^{T} A_{1 i} & P^{T} B_{i} \\
* & -(1-\mu) Q & 0 \\
* & * & -\gamma^{2} I
\end{array}\right]<0
\end{gathered}
$$

then, system (40) is asymptotically stable for $w(t)=0$ and $\|z(t)\|_{2}<\gamma\|w(t)\|_{2}$ for zero initial conditions.

Proof: The following Lyapunov-Krasovskii functional is considered:

$V\left(t, x_{t}\right)=x^{T}(t) P^{T} E x(t)+\int_{t-\tau(t)}^{t} x^{T}(\lambda) Q x(\lambda) d \lambda$

in which $P^{T} E=E^{T} P \geq 0, Q=Q^{T}>0$ and $x_{t}:=x(t+$ $\omega)$ where $\omega \in\left[-\tau_{m}, 0\right]$. Consider the index:

$$
J=\int_{0}^{\infty}\left[z(t)^{T} z(t)-\gamma^{2} w(t)^{T} w(t)\right] d t .
$$

Showing that $J<0$ for zero initial condition case and $\dot{V}\left(t, x_{t}\right)<0$ for zero input case proves the lemma. $J$ can be written as follows:

$$
\begin{aligned}
J= & \int_{0}^{\infty}\left[z(t)^{T} z(t)-\gamma^{2} w(t)^{T} w(t)+\dot{V}\left(t, x_{t}\right)\right] d t \\
& +\left.V\left(t, x_{t}\right)\right|_{t=0}-\left.V\left(t, x_{t}\right)\right|_{t=\infty}
\end{aligned}
$$

in which $\left.V\left(t, x_{t}\right)\right|_{t=0}=0$ and $\left.V\left(t, x_{t}\right)\right|_{t=\infty} \geq 0$, so:

$$
J \leq \int_{0}^{\infty}\left[z(t)^{T} z(t)-\gamma^{2} w(t)^{T} w(t)+\dot{V}\left(t, x_{t}\right)\right] d t .
$$

Considering the convex property of weighting functions (4) and the maximum bound of delay derivative $(\mu)$ :

$$
J \leq \int_{0}^{\infty} \sum_{i=1}^{h} \rho_{i}(\theta(t)) \zeta(t)^{T} \Theta^{i} \zeta(t) d t
$$

where $\zeta(t)=\left[x(t)^{T} x(t-\tau(t))^{T} w(t)^{T}\right]^{T}$. So, $\Theta^{i}<0$ assures $J<0$. The asymptotic stability of (40) is deduced from negative definiteness of the following submatrix of $\Theta^{i}$ :

$$
\left[\begin{array}{cc}
P^{T} A_{0 i}+A_{0 i}^{T} P+Q & P^{T} A_{1 i} \\
* & -(1-\mu) Q
\end{array}\right]
$$

which results in $\dot{V}\left(t, x_{t}\right)<0$ in the non-actuated case.

Remark 4.3: The robust stability criterion presented in Lemma 4.1 is a delay derivative dependent and delay independent condition. This Lemma will be used in the forthcoming parts to derive the UIO design procedure in LMI format. A delay dependent condition for singular delayed LPV systems' robust stability has been presented in Li and Zhang (2012) but using that condition to guarantee the convergence of the UIO needs resolving some nonlinearities in the matrix inequalities which is beyond the scope of the current study.

Lemma 4.2: (Yang, Wang, Hung, \& Shu, 2005): Let $M, N$ and $\Pi$ be real matrices with appropriate dimensions and matrix $\Sigma(t)$ satisfying $\Sigma(t)^{T} \Sigma(t) \leq I$, then

$$
\Pi+M \Sigma N+N^{T} \Sigma^{T} M^{T}<0
$$

if and only if there exists a positive scalar $\varepsilon$ such that

$$
\Pi+\frac{1}{\varepsilon} M M^{T}+\varepsilon N^{T} N<0
$$

\subsection{Main results}

Using the material introduced so far, the following Theorem can be stated.

Theorem 4.1: Considering system (7), if there exist symmetric positive definite matrices $P_{2}, Q_{1}$ and $Q_{2}$, matrices $P_{1}$, $M$ and $M_{\text {si }}$ for $s=0,1$ and $i=1, \ldots, h$ and positive scalars $\bar{\gamma}, \varepsilon_{A_{0}}, \varepsilon_{A_{1}}$ and $\varepsilon_{B}$ obtained from the solution to the following optimisation problem:

$$
\min _{P_{1}, P_{2}, Q_{1}, Q_{2}, M, M_{s i}, \varepsilon_{A_{0}}, \varepsilon_{A_{1}}, \varepsilon_{B}} \bar{\gamma}
$$

subject to the following LMI constraints for $i=1, \ldots, h$ :

$$
P_{1}^{T} \tilde{E}=\tilde{E}^{T} P_{1} \geq 0
$$




$$
\Omega^{i}=\left[\begin{array}{ccc}
\Omega_{11}^{i} & \cdots & \Omega_{19}^{i} \\
* & \ddots & \vdots \\
* & * & \Omega_{99}^{i}
\end{array}\right]<0
$$

where

$$
\begin{gathered}
\Omega_{11}^{i}=\left[\begin{array}{cc}
\operatorname{sym}\left\{P_{1}^{T} \tilde{A}_{0 i}\right\}+Q_{1} & 0 \\
0 & \operatorname{sym}\left\{P_{2}^{T} H_{10} \tilde{A}_{0 i}+M V_{1} \tilde{A}_{0 i}-M_{0 i} \tilde{C}\right\}+I+Q_{2}
\end{array}\right] \\
\Omega_{12}^{i}=\left[\begin{array}{cc}
P_{1}^{T} \tilde{A}_{1 i} & 0 \\
0 & P_{2}^{T} H_{10} \tilde{A}_{1 i}+M V_{1} \tilde{A}_{1 i}-M_{1 i} \tilde{C}
\end{array}\right] \\
\Omega_{22}^{i}=\left[\begin{array}{cc}
-(1-\mu) Q_{1} & 0 \\
0 & -(1-\mu) Q_{2}
\end{array}\right]
\end{gathered}
$$$$
\Omega_{13}^{i}=\left[\begin{array}{ccccc}
P_{1}^{T} \tilde{B}_{i} P_{1}^{T} \tilde{R}_{i} P_{1}^{T} \tilde{F} & 0 & 0 & 0 \\
0 & 0 & 0 & -M_{0 i} D_{n}-M_{1 i} D_{n}-P_{2}^{T} H_{20} D_{n}-M V_{2} D_{n}
\end{array}\right],
$$$$
\Omega_{23}^{i}=0, \Omega_{33}^{i}=-\bar{\gamma} I \Omega_{14}^{i}=\left[\begin{array}{ccc}
0 & 0 \\
{\left[\begin{array}{ccc}
\Gamma_{01} & \cdots & \Gamma_{0 h}
\end{array}\right]-\left[\begin{array}{ccc}
\bar{\Gamma}_{01} & \cdots & \bar{\Gamma}_{0 h}
\end{array}\right]}
\end{array}\right]
$$$$
\Gamma_{0 j}=P_{2}^{T} H_{10} \tilde{A}_{0 j}+M V_{1} \tilde{A}_{0 j},
$$$$
\bar{\Gamma}_{0 j}=P_{2}^{T} H_{10} \tilde{A}_{0 j}+M V_{1} \tilde{A}_{0 j}-M_{0 j} \tilde{C} \quad(\text { for } \quad j=1, \ldots, h)
$$$$
\Omega_{24}^{i}=0, \Omega_{34}^{i}=0, \Omega_{44}^{i}=-\varepsilon_{A_{0}} I
$$$$
\Omega_{15}^{i}=\left[\begin{array}{cc}
\varepsilon_{A_{0}} \phi_{x}^{T} & 0 \\
0 & \varepsilon_{A_{0}} \phi_{x}^{T}
\end{array}\right], \Omega_{25}^{i}=0, \Omega_{35}^{i}=0, \Omega_{45}^{i}=0,
$$$$
\Omega_{55}^{i}=-\varepsilon_{A_{0}} I
$$$$
\Omega_{16}^{i}=\left[\begin{array}{cc}
0 & 0 \\
{\left[\Gamma_{11} \cdots \Gamma_{1 h}\right]-\left[\bar{\Gamma}_{11} \cdots \bar{\Gamma}_{1 h}\right]}
\end{array}\right]
$$$$
\Gamma_{1 j}=P_{2}^{T} H_{10} \tilde{A}_{1 j}+M V_{1} \tilde{A}_{1 j} \text {, }
$$$$
\bar{\Gamma}_{1 j}=P_{2}^{T} H_{10} \tilde{A}_{1 j}+M V_{1} \tilde{A}_{1 j}-M_{1 j} \tilde{C} \quad(\text { for } \quad j=1, \ldots, h)
$$$$
\Omega_{26}^{i}=0, \Omega_{36}^{i}=0, \Omega_{46}^{i}=0, \Omega_{56}^{i}=0, \Omega_{66}^{i}=-\varepsilon_{A_{1}} I
$$$$
\Omega_{17}^{i}=0, \quad \Omega_{27}^{i}=\left[\begin{array}{cc}
\varepsilon_{A_{1}} \phi_{x}^{T} & 0 \\
0 & \varepsilon_{A_{1}} \phi_{x}^{T}
\end{array}\right], \quad \Omega_{37}^{i}=0, \quad \Omega_{47}^{i}=0,
$$$$
\Omega_{57}^{i}=0, \quad \Omega_{67}^{i}=0, \quad \Omega_{77}^{i}=-\varepsilon_{A_{1}} I
$$

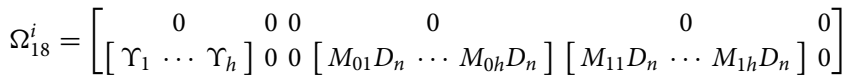

$$
\begin{aligned}
& \Upsilon_{j}=P_{2}^{T} H_{10} \tilde{B}_{j}+M V_{1} \tilde{B}_{j} \quad(\text { for } \quad j=1, \ldots, h) \\
& \Omega_{28}^{i}=0, \quad \Omega_{38}^{i}=0, \quad \Omega_{48}^{i}=0, \quad \Omega_{58}^{i}=0, \\
& \Omega_{68}^{i}=0, \quad \Omega_{78}^{i}=0, \quad \Omega_{88}^{i}=-\varepsilon_{B} I
\end{aligned}
$$

$\Omega_{19}^{i}=0, \quad \Omega_{29}^{i}=0, \quad \Omega_{39}^{i}=\operatorname{diag}\left(\varepsilon_{B} \phi_{u}^{T}, \varepsilon_{B} \phi_{d}^{T}, \varepsilon_{B} \phi_{f}^{T}, \varepsilon_{B} \phi_{n}^{T}, \varepsilon_{B} \phi_{n}^{T}, \varepsilon_{B} \bar{\phi}_{n}^{T}\right)$ $\Omega_{49}^{i}=0, \quad \Omega_{59}^{i}=0, \quad \Omega_{69}^{i}=0, \quad \Omega_{79}^{i}=0, \quad \Omega_{89}^{i}=0, \quad \Omega_{99}^{i}=-\varepsilon_{B} I$

then, the robust state and fault estimator (8) with the attenuation level $\gamma=\sqrt{\gamma}$ exists and by means of the following variables:

$$
\begin{aligned}
K & =P_{2}^{-1} M \\
K_{s i} & =P_{2}^{-1} M_{s i} \quad \text { for } s=0,1 \text { and } i=1, \ldots, h,
\end{aligned}
$$

the UIO matrices $G_{i}, H_{2}, N_{s i}$ and $L_{s i}$ can be calculated from (21), (39), (26) and (25), respectively.

Proof: System (30) is considered with the output defined as $z(t)=\bar{C} \xi(t)=\left[\begin{array}{ll}0 & I_{n}\end{array}\right] \xi(t)=e(t)$. Lemma 4.1 is used to guarantee the robust stability of this system. Applying the conditions of Lemma 4.1 on uncertain system (30) results in:

$$
\begin{aligned}
& P^{T} \bar{E}=\bar{E}^{T} P \geq 0 \\
& \Pi_{i}=\left[\begin{array}{ccc}
\operatorname{sym}\left\{P^{T}\left(\bar{A}_{0 i}+\Delta \bar{A}_{0 i}\right)\right\}+Q+\bar{C}^{T} \bar{C} P^{T}\left(\bar{A}_{1 i}+\Delta \bar{A}_{1 i}\right) & P^{T}\left(\bar{B}_{i}+\Delta \bar{B}_{i}\right) \\
* & -(1-\mu) Q & 0 \\
* & * & -\gamma^{2} I
\end{array}\right] \\
& <0
\end{aligned}
$$

which can be transformed into:

$\Pi_{i}=\Pi_{i}^{0}+\operatorname{sym}\left\{\Pi_{i}^{\Delta A_{0}}\right\}+\operatorname{sym}\left\{\Pi_{i}^{\Delta A_{1}}\right\}+\operatorname{sym}\left\{\Pi_{i}^{\Delta B}\right\}<0$

where

$$
\begin{aligned}
& \Pi_{i}^{0}=\left[\begin{array}{ccc}
P^{T} \bar{A}_{0 i}+\bar{A}_{0 i}^{T} P+Q+\bar{C}^{T} \bar{C} & P^{T} \bar{A}_{1 i} & P^{T} \bar{B}_{i} \\
* & -(1-\mu) Q & 0 \\
* & * & -\gamma^{2} I
\end{array}\right], \\
& \Pi_{i}^{\Delta A_{0}}=\left[\begin{array}{cccc}
P^{T} \Delta \bar{A}_{0 i} & 0 & 0 \\
* & 0 & 0 \\
* & * & 0
\end{array}\right], \Pi_{i}^{\Delta A_{1}}=\left[\begin{array}{ccc}
0 & P^{T} \Delta \bar{A}_{1 i} & 0 \\
* & 0 & 0 \\
* & * & 0
\end{array}\right], \\
& \Pi_{i}^{\Delta B}=\left[\begin{array}{lll}
0 & 0 & P^{T} \Delta \bar{B}_{i} \\
* & 0 & 0 \\
* & * & 0
\end{array}\right]
\end{aligned}
$$

Considering (31)-(33), (57) transforms into:

$$
\begin{aligned}
\Pi_{i}= & \Pi_{i}^{0}+\operatorname{sym}\left\{\tilde{M}_{A_{0}} \Sigma_{A}(t) \tilde{N}_{A_{0}}\right\}+\operatorname{sym}\left\{\tilde{M}_{A_{1}} \Sigma_{A}(t) \tilde{N}_{A_{1}}\right\} \\
& +\operatorname{sym}\left\{\tilde{M}_{B} \Sigma_{B}(t) \tilde{N}_{B}\right\}<0
\end{aligned}
$$

where

$$
\begin{aligned}
& \tilde{M}_{A_{0}}=\left[\begin{array}{c}
P^{T} M_{A_{0}} \\
0 \\
0
\end{array}\right], \tilde{M}_{A_{1}}=\left[\begin{array}{c}
P^{T} M_{A_{1}} \\
0 \\
0
\end{array}\right], \tilde{M}_{B}=\left[\begin{array}{c}
P^{T} M_{B} \\
0 \\
0
\end{array}\right], \\
& \tilde{N}_{A_{0}}=\left[\begin{array}{lll}
N_{A} & 0
\end{array}\right], \tilde{N}_{A_{1}}=\left[\begin{array}{lll}
0 & N_{A} & 0
\end{array}\right], \tilde{N}_{B}=\left[\begin{array}{lll}
0 & 0 & N_{B}
\end{array}\right]
\end{aligned}
$$

Due to properties (34) -(35) and by applying Lemma 4.2, (58) can be rewritten as follows:

$$
\begin{gathered}
\Pi_{i}^{0}+\frac{1}{\varepsilon_{A_{0}}} \tilde{M}_{A_{0}} \tilde{M}_{A_{0}}^{T}+\varepsilon_{A_{0}} \tilde{N}_{A_{0}}^{T} \tilde{N}_{A_{0}}+\frac{1}{\varepsilon_{A_{1}}} \tilde{M}_{A_{1}} \tilde{M}_{A_{1}}^{T} \\
+\varepsilon_{A_{1}} \tilde{N}_{A_{1}}^{T} \tilde{N}_{A_{1}}+\frac{1}{\varepsilon_{B}} \tilde{M}_{B} \tilde{M}_{B}^{T}+\varepsilon_{B} \tilde{N}_{B}^{T} \tilde{N}_{B}<0
\end{gathered}
$$


and then by applying the Schur complement Lemma, (59) results in:

$$
\left[\begin{array}{ccccccc}
\Pi_{i}^{0} & \tilde{M}_{A_{0}} & \varepsilon_{A_{0}} \tilde{N}_{A_{0}}^{T} & \tilde{M}_{A_{1}} & \varepsilon_{A_{1}} \tilde{N}_{A_{1}}^{T} & \tilde{M}_{B} & \varepsilon_{B} \tilde{N}_{B}^{T} \\
* & -\varepsilon_{A_{0}} I & 0 & 0 & 0 & 0 & 0 \\
* & * & -\varepsilon_{A_{0}} I & 0 & 0 & 0 & 0 \\
* & * & * & -\varepsilon_{A_{1}} I & 0 & 0 & 0 \\
* & * & * & * & -\varepsilon_{A_{1}} I & 0 & 0 \\
* & * & * & * & * & -\varepsilon_{B} I & 0 \\
* & * & * & * & * & * & -\varepsilon_{B} I
\end{array}\right]<0 .
$$

Now, by choosing the following matrix blocks as Lyapunov-Krasovskii matrices when using Lemma 4.1:

$$
P=\left[\begin{array}{cc}
P_{1} & 0 \\
0 & P_{2}
\end{array}\right] Q=\left[\begin{array}{cc}
Q_{1} & 0 \\
0 & Q_{2}
\end{array}\right]
$$

the condition $P^{T} \bar{E}=\bar{E}^{T} P \geq 0$ is equivalent with (51) and $P_{2}=P_{2}^{T} \geq 0$. Substituting the corresponding terms of $\Pi_{i}^{0}, \tilde{M}_{A_{0}}, \tilde{N}_{A_{0}}, \tilde{M}_{A_{1}}, \tilde{N}_{A_{1}}, \tilde{M}_{B}$ and $\tilde{N}_{B}$ based on the matrices of augmented system (30) in (60) and then by applying the following change of variables to resolve the nonlinearities in the matrix inequality obtained

$$
\begin{aligned}
M & :=P_{2} K \\
M_{s i}:=P_{2} K_{s i} \quad \text { for } s= & 0,1 \text { and } i=1, \ldots, h \\
\bar{\gamma} & :=\gamma^{2},
\end{aligned}
$$

the LMI (52) is derived. Whenever the optimisation problem (50) under LMIs (51)-(52) is solved, $K$ and $K_{s i}$ are, respectively, obtained by (53) -(54) which result from (61) to (62). The unknown matrices of UIO (8) are then calculated from (21), (39), (26) and (25). So, the robust convergence of the state and fault estimator (8) with the unknown input attenuation level $\gamma=\sqrt{\bar{\gamma}}$ is achieved considering Lemma 4.1. This ends the proof.

Remark 4.4: Theorem 4.1 has the constraint $P_{1}^{T} \tilde{E}=$ $\tilde{E}^{T} P_{1} \geq 0$ in its formulation which may result in numerical problems. To avoid these problems, this constraint can be considered by parameter ising $P_{1}$ as $\mathcal{P}_{1}=P_{1} \tilde{E}+S X$ where $P_{1}>0$ and $X \in R^{(n-r) \times n}$ are the parameter matrices and $S \in R^{n \times(n-r)}$ is any full column rank matrix which satisfies $\tilde{E}^{T} S=0$ (Lam \& Xu, 2006).

Corollary 4.1: Considering the system (7), if there exist symmetric positive definite matrices $P_{1}, P_{2}, Q_{1}$ and $Q_{2}$, matrices $X, M$ and $M_{s i}$ for $s=0,1$ and $i=1, \ldots, h$ and positive scalars $\bar{\gamma}, \varepsilon_{A_{0}}, \varepsilon_{A_{1}}$ and $\varepsilon_{B}$ obtained as the solution to the following optimisation problem:

$$
\min _{P_{1}, P_{2}, Q_{1}, Q_{2}, X, M, M_{s i}, \varepsilon_{A_{0}}, \varepsilon_{A_{1}}, \varepsilon_{B}} \bar{\gamma}
$$

subject to the following LMI constraints for $i=1, \ldots, h$ :

$$
\tilde{\Omega}^{i}=\left[\begin{array}{ccc}
\tilde{\Omega}_{11}^{i} & \cdots & \tilde{\Omega}_{19}^{i} \\
* & \ddots & \vdots \\
* & * & \tilde{\Omega}_{99}^{i}
\end{array}\right]<0
$$

where all blocks of $\tilde{\Omega}^{i}$ are equal with the corresponding blocks of $\Omega^{i}$ defined in Theorem 4.1 except the blocks $\tilde{\Omega}_{1 \tilde{E}}^{i}, \tilde{\Omega}_{12}^{i}$ and $\tilde{\Omega}_{13}^{i}$ in which $P_{1}$ is substituted with $\mathcal{P}_{1}=$ $P_{1} \tilde{E}+S X$ and $S \in R^{n \times(n-r)}$ is any full column rank matrix which satisfies $\tilde{E}^{T} S=0$. Then, the robust state and fault estimator (8) with the unknown input attenuation level $\gamma=\sqrt{\bar{\gamma}}$ exists. The matrices $K$ and $K_{s i}$ are obtained by (53) - (54) and the UIO matrices $G_{i}, H_{2}, N_{s i}$ and $L_{s i}$ are then calculated, respectively from (21), (39), (26) and (25).

Remark 4.5: The formulation in this paper is derived for the singular delayed LPV systems with inexact measured parameters. However, the results can also be used for polytopic LPV systems with unmeasurable scheduling functions as in Theilliol and Aberkane (2011) and LópezEstrada et al. (2014). In this case, systems (5) and (7) are scheduled according to $\rho_{i}(x(t))$ and UIO (8) is scheduled according to $\rho_{i}(\hat{x}(t))$. Considering the substitution of $\rho_{i}(\theta(t)), \rho_{i}(\hat{\theta}(t)), \rho_{j}(\theta(t))$ and $\rho_{j}(\hat{\theta}(t))$, respectively, with $\rho_{i}(x(t)), \rho_{i}(\hat{x}(t)), \rho_{j}(x(t))$ and $\rho_{j}(\hat{x}(t))$ in the corresponding equations, the results obtained are also valid for the singular delayed LPV systems with unmeasurable scheduling variables.

\subsection{Fault diagnosis}

The UIO (8) designed in this paper can be used for state estimation and fault diagnosis in singular delayed LPV systems with inexact parameters which can include both cases of inexact measured parameters and unmeasurable parameters. The state estimation of this UIO is valid both in fault free and faulty situations in comparison to residual-based approaches for fault detection in which state estimates are just valid in fault free conditions and they deviate from true values in faulty conditions. Thus, this UIO could be used for implementing an observer based fault tolerant controller.

Based on this UIO, fault diagnosis is obtained directly via estimating the size of faults in the system. The advantage of this method is that unlike the common observerbased fault detection methods, it is not needed to first generate residuals and then evaluate them in order to detect the possible faults in the system. Furthermore, for the fault isolation phase, it is not needed to design a bank of observers as being discussed in Hassanabadi et al. (2016b), because each fault is automatically isolated when 

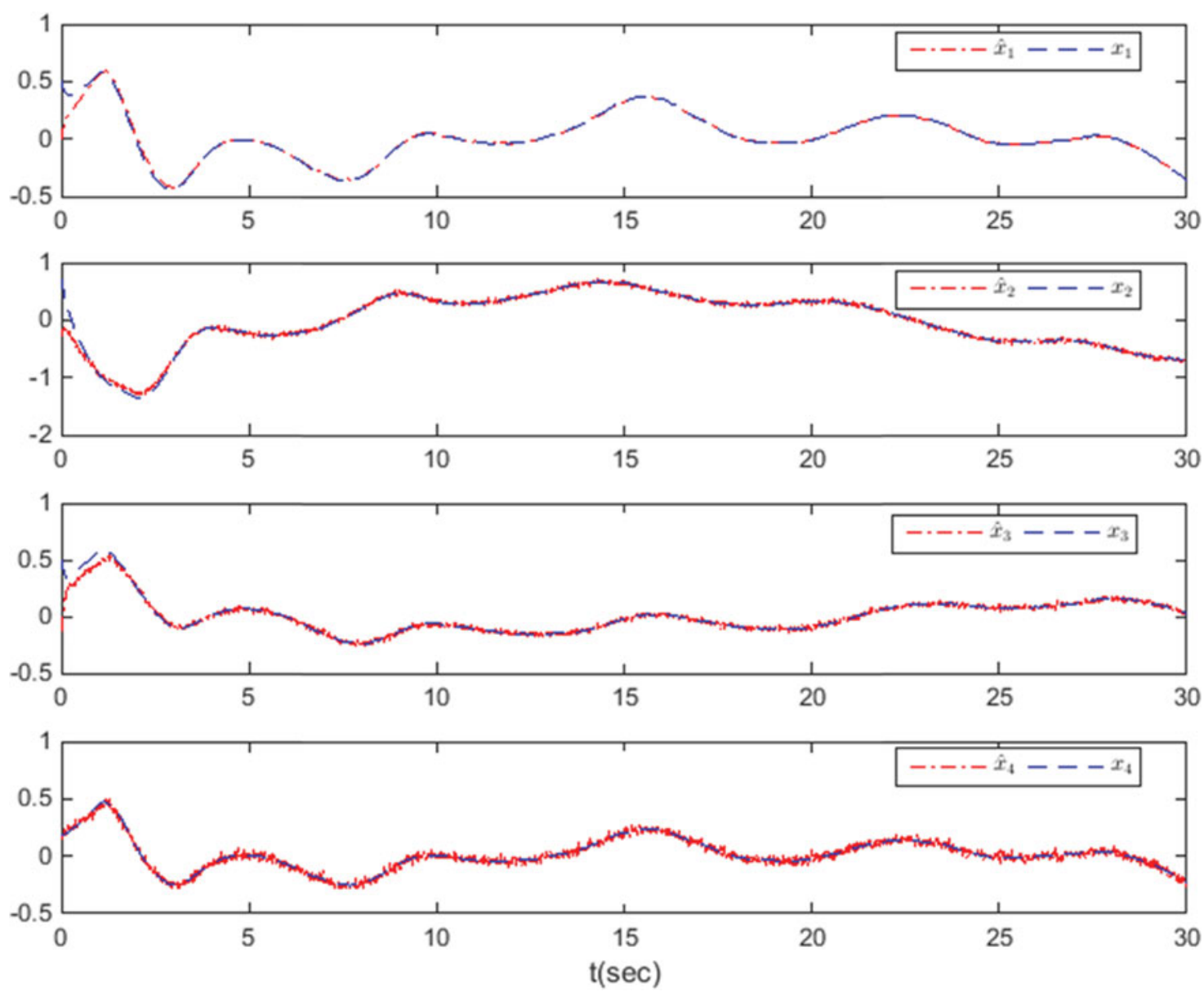

Figure 1. System states and their estimates in the first scenario.

its estimate starts to have a significant deviation from zero. Thus, fault estimation (last step of fault diagnosis) is also automatically obtained.

Remark 4.6: Further research can be conducted for fault diagnosis of discrete-time singular delayed LPV systems based on discrete-time version of the UIO proposed in this paper and the stability results proposed in Zhang and Zhu (2012).

\subsection{Summary of the method}

Algorithm 1. Robust state and fault estimation system design for singular delayed LPV systems with inexact parameters

\section{Illustrative example}

\subsection{Description}

An example is used to illustrate the performance of the proposed state and fault estimation method. System (1) with the following numerical values is considered:

\section{Algorithm 1}

Step 0. Check Assumption 2.1 and condition (37).

Step 1. Calculate $H_{10}=\Psi Y_{1}{ }^{+}, H_{20}=\Psi Y_{2}{ }^{+}, V_{1}=V T_{1}$ and $V_{2}=V T_{2}$. Step 2. Solve the convex optimisation problem (64) and obtain matrices $P_{1}, P_{2}, M, X, Q_{1}, Q_{2}$ and $M_{s i}$ (for $i=1, \ldots, h$ and $s=0,1$ ). Step 3. Calculate $K$ and $K_{s i}$ (for $i=1, \ldots, h$ and $s=0,1$ ) from (53) and (54), respectively.

Step 4. Calculate $H_{1}$ and $H_{2}$ from (39).

Step 5. Calculate $N_{s i}$ (for $i=1, \ldots, h$ and $s=0,1$ ) and $G_{i}$ from (26) and (21), respectively.

Step 6. Calculate $L_{s i}$ (for $i=1, \ldots, h$ and $s=0,1$ ) from (25).

$$
\begin{aligned}
E= & {\left[\begin{array}{llll}
1 & 0 & 0 & 0 \\
0 & 1 & 0 & 0 \\
0 & 0 & 1 & 0 \\
0 & 0 & 0 & 0
\end{array}\right], } \\
A_{0}(\theta(t)) & =,
\end{aligned}
$$$$
=\left[\begin{array}{cccc}
2.2+0.1 \theta_{1}(\mathrm{t}) & -1.3 & -7.8 & 1.7+0.5 \theta_{2}(\mathrm{t}) \\
6.1 & -2.8 & -11.8-0.3 \theta_{1}(\mathrm{t}) & 0.5 \\
9.2 & -2.6-0.2 \theta_{1}(\mathrm{t}) & -16.7 & -0.7 \\
12.8 & -1.9 & -4.1-0.1 \theta_{2}(\mathrm{t}) & -14.4
\end{array}\right] \text {, }
$$

$A_{1}(\theta(t))=\left[\begin{array}{cccc}0 & 1+0.2 \theta_{1}(t) & 0 & -0.5 \\ -1.2 & 0 & -1+0.4 \theta_{2}(t) & 0.8 \\ 0 & 1-0.1 \theta_{1}(t) & 0 & 0 \\ -0.7 & 0 & 0.3 & 0\end{array}\right]$, 

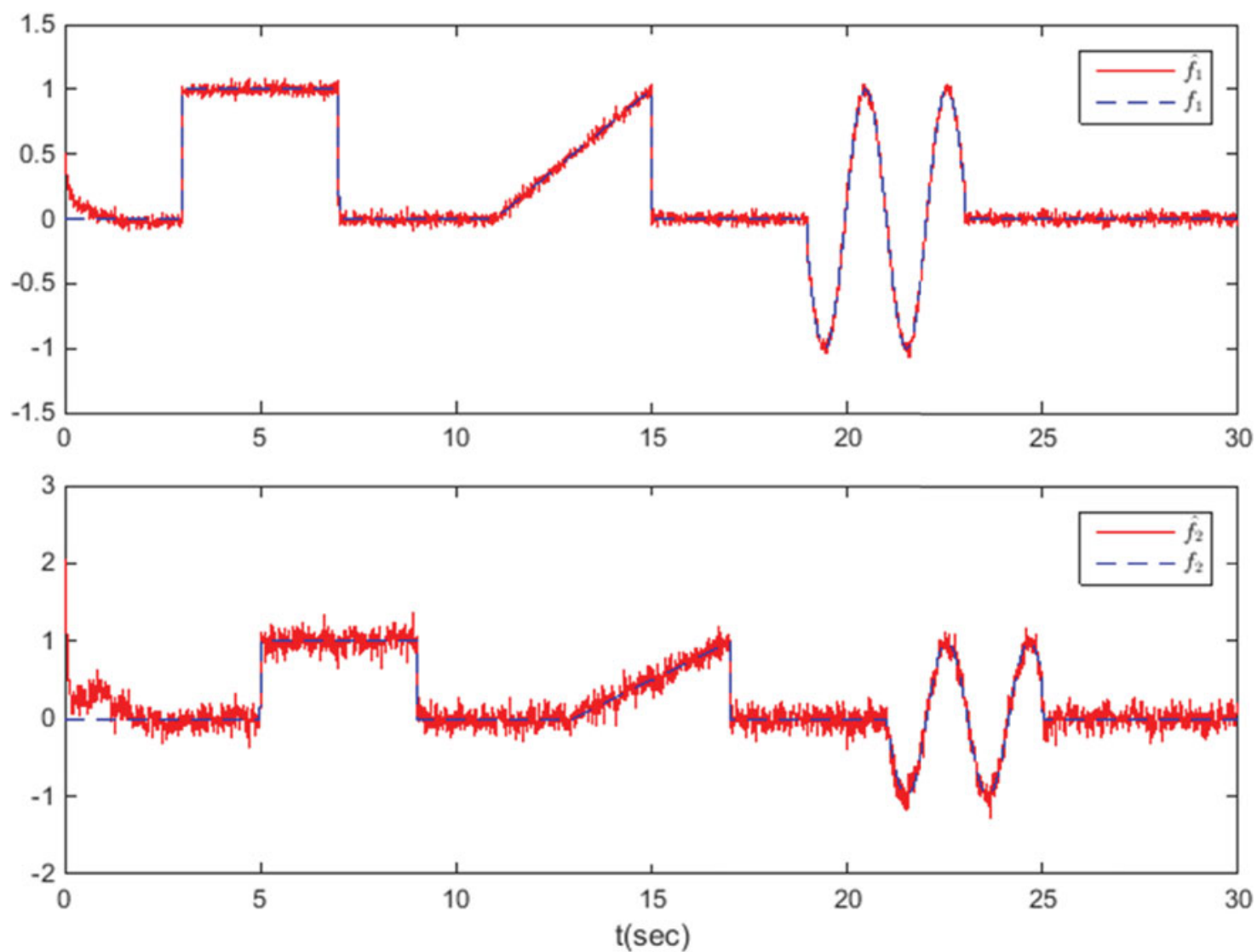

Figure 2. Estimation of the faults on the first and the second sensor in the first scenario.

$$
\begin{aligned}
& B(\theta(t))= {\left[\begin{array}{c}
1+0.15 \theta_{1}(t) \\
0.5 \\
2+0.25 \theta_{1}(t) \\
0 \\
R
\end{array}\right]\left[\begin{array}{c}
0 \\
1 \\
0.5 \\
0
\end{array}\right], C=\left[\begin{array}{cccc}
-1 & 1 & 0 & 2 \\
4 & -2 & 3 & 1 \\
1 & 0 & -1 & 5 \\
-2 & 1 & 0 & 1
\end{array}\right], } \\
& D_{f}=\left[\begin{array}{ll}
1 & 0 \\
0 & 1 \\
0 & 0 \\
0 & 0
\end{array}\right], D_{n}=\left[\begin{array}{c}
0.5 \\
-0.7 \\
1.2 \\
0
\end{array}\right]
\end{aligned}
$$

The delay is $\tau(t)=0.6+0.4 \sin (t)$ seconds. The range of the two parameters are $\theta_{1}(t) \in[-1,1]$ and $\theta_{2}(t) \in$ $[-1.5,1.5]$. In this case, the polytopic representation (5) consists of four subsystems defined in one of the vertices of the parameter variation domain. The gain matrices of theses subsystems are calculated as follows:

$$
X_{1}=\left.X(\theta(t))\right|_{\substack{\theta_{1}(t)=\theta_{1}^{m} \\ \theta_{2}(t)=\theta_{2}^{m}}}, \quad X_{2}=\left.X(\theta(t))\right|_{\substack{\theta_{1}(t)=\theta_{1}^{M} \\ \theta_{2}(t)=\theta_{2}^{m}}},
$$

$$
X_{3}=\left.X(\theta(t))\right|_{\substack{\theta_{1}(t)=\theta_{1}^{M} \\
\theta_{2}(t)=\theta_{2}^{M}}}, \quad X_{4}=\left.X(\theta(t))\right|_{\begin{array}{r}
\theta_{1}(t)=\theta_{1}^{m} \\
\theta_{2}(t)=\theta_{2}^{M}
\end{array}}
$$

where $X$ represents $A_{0}, A_{1}$ and $B . X_{i}$ for $i=1, \ldots, 4$ is the corresponding matrix in the subsystem $i$. The corresponding time-varying weights of the four subsystems of the polytopic singular delayed LPV system (5) are calculated as follows:

$$
\begin{aligned}
\rho_{1}(\theta(t)) & =\alpha_{1}(t) \alpha_{2}(t), \quad \rho_{2}(\theta(t))=\left(1-\alpha_{1}(t)\right) \alpha_{2}(t) \\
\rho_{3}(\theta(t)) & =\left(1-\alpha_{1}(t)\right)\left(1-\alpha_{2}(t)\right), \quad \rho_{4}(\theta(t)) \\
& =\alpha_{1}(t)\left(1-\alpha_{2}(t)\right)
\end{aligned}
$$

where $\alpha_{1}(t)=\left(\theta_{1}^{M}-\theta_{1}(t)\right) /\left(\theta_{1}^{M}-\theta_{1}^{m}\right)$ and $\alpha_{2}(t)=$ $\left(\theta_{2}^{M}-\theta_{2}(t)\right) /\left(\theta_{2}^{M}-\theta_{2}^{m}\right)$.

\subsection{Results}

Now, a UIO in the form of (8) is designed based on Algorithm 1. The convex optimisation (64) is solved with the SeDuMi solver (Sturm, 1999) using the YALMIP toolbox (Löfberg, 2004). The obtained UIO matrices 

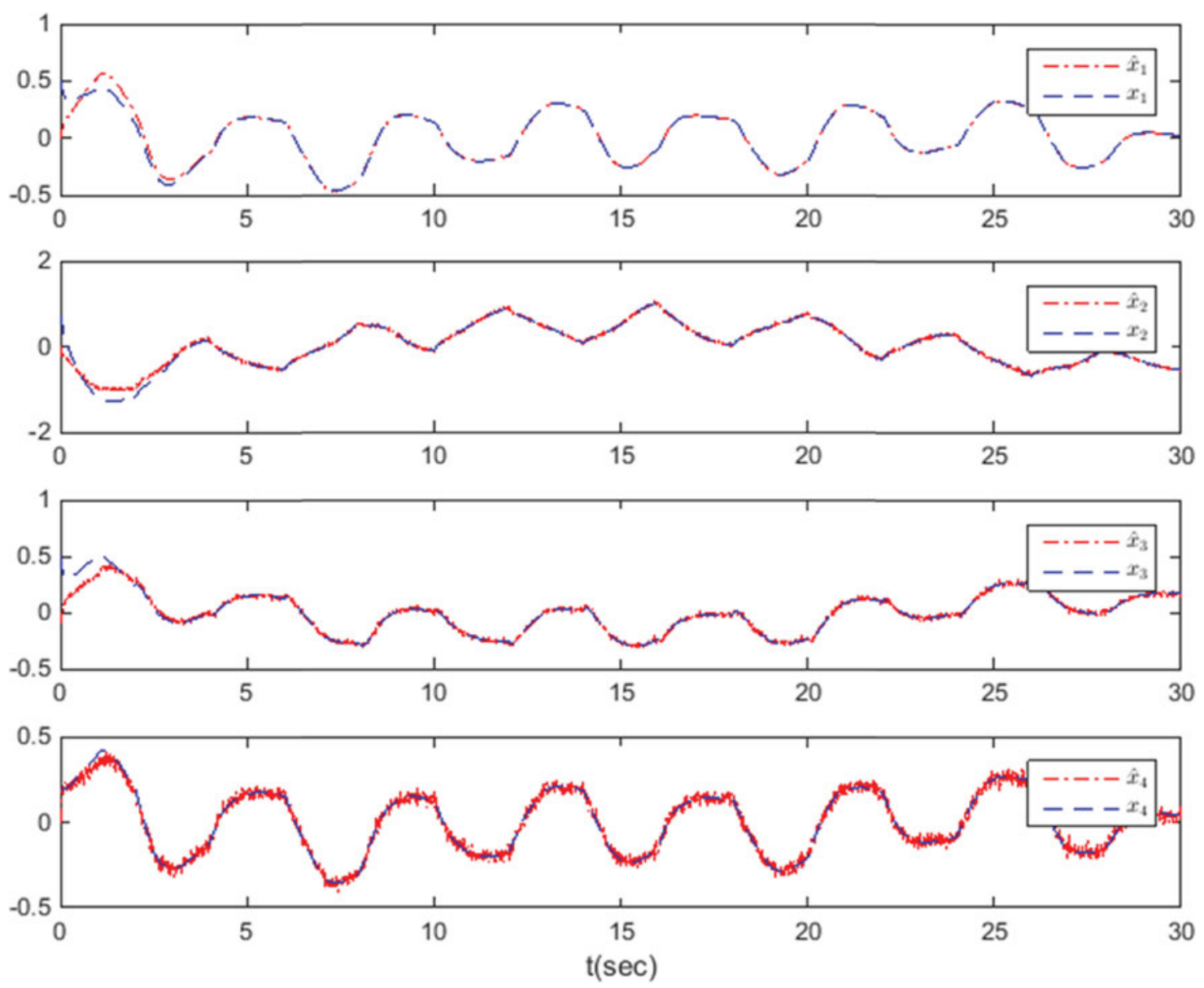

Figure 3. System states and their estimates in the second scenario.

are:

$$
\begin{aligned}
& H_{2}=\left[\begin{array}{cccc}
0.0000 & 0.0000 & -0.0000 & -0.0000 \\
0.0000 & 0.0000 & -0.1818 & 0.9091 \\
0.0000 & 0.0000 & -0.0909 & 0.4545 \\
0.0000 & 0.0000 & 0.1818 & 0.0909 \\
1.0000 & -0.0000 & -0.1818 & -1.0909 \\
-0.0000 & 1.0000 & -0.2727 & 0.3636
\end{array}\right] \text {, } \\
& G_{1}=\left[\begin{array}{c}
0.8500 \\
1.4500 \\
2.1000 \\
0.2500 \\
-1.1000 \\
-7.0500
\end{array}\right], G_{2}=\left[\begin{array}{c}
1.1500 \\
2.0500 \\
2.4000 \\
0.2500 \\
-1.4000 \\
-7.9500
\end{array}\right] \text {, } \\
& G_{3}=\left[\begin{array}{c}
1.1500 \\
1.9136 \\
3.0818 \\
0.3864 \\
-1.5364 \\
-10.4045
\end{array}\right], G_{4}=\left[\begin{array}{c}
0.8500 \\
1.3136 \\
2.7818 \\
0.3864 \\
-1.2364 \\
-9.5045
\end{array}\right]
\end{aligned}
$$

Although there are four subsystems, due to space limitations, only the results of the first subsystem $(i=1)$ for the matrices $N_{0 i}, N_{1 i}, L_{0 i}$ and $L_{1 i}$ are presented here:

$$
\begin{aligned}
& N_{01}=\left[\begin{array}{cccccc}
-0.5748 & -1.0027 & -0.2694 & -0.1468 & 0.2885 & 1.8551 \\
-1.1054 & -1.9674 & -0.4884 & -0.2434 & 0.5847 & 3.2651 \\
-0.7958 & -1.1933 & -0.5217 & -0.3982 & 0.2501 & 4.0811 \\
-0.0442 & -0.0381 & -0.0504 & -0.0503 & -0.0077 & 0.4452 \\
0.6190 & 1.0408 & 0.3199 & 0.1971 & -0.2808 & -2.3003 \\
2.5199 & 3.6942 & 1.7163 & 1.3456 & -0.7273 & -13.5787
\end{array}\right] \\
& N_{11}=\left[\begin{array}{cccccc}
0.2886 & 0.6303 & 0.0339 & -0.0531 & -0.2399 & 0.0637 \\
0.4823 & 1.0557 & 0.0448 & -0.0911 & -0.3988 & 0.1496 \\
0.7632 & 1.6542 & 0.1485 & -0.1279 & -0.6456 & -0.0476 \\
0.0949 & 0.2048 & 0.0229 & -0.0150 & -0.0811 & -0.0223 \\
-0.3835 & -0.8351 & -0.0568 & 0.0680 & 0.3211 & -0.0414 \\
-2.5743 & -5.5771 & -0.5143 & 0.4285 & 2.1801 & 0.2096
\end{array}\right] \\
& L_{01}=\left[\begin{array}{cccc}
-0.0000 & -0.0000 & 1.2711 & -4.2547 \\
-0.0000 & -0.0000 & 2.2602 & -7.6745 \\
-0.0000 & -0.0000 & 2.6814 & -8.4293 \\
-0.0000 & -0.0000 & 0.2821 & -0.8349 \\
0.0000 & 0.0000 & -1.5532 & 5.0896 \\
0.0000 & 0.0000 & -8.8905 & 27.7928
\end{array}\right] \text {, }
\end{aligned}
$$



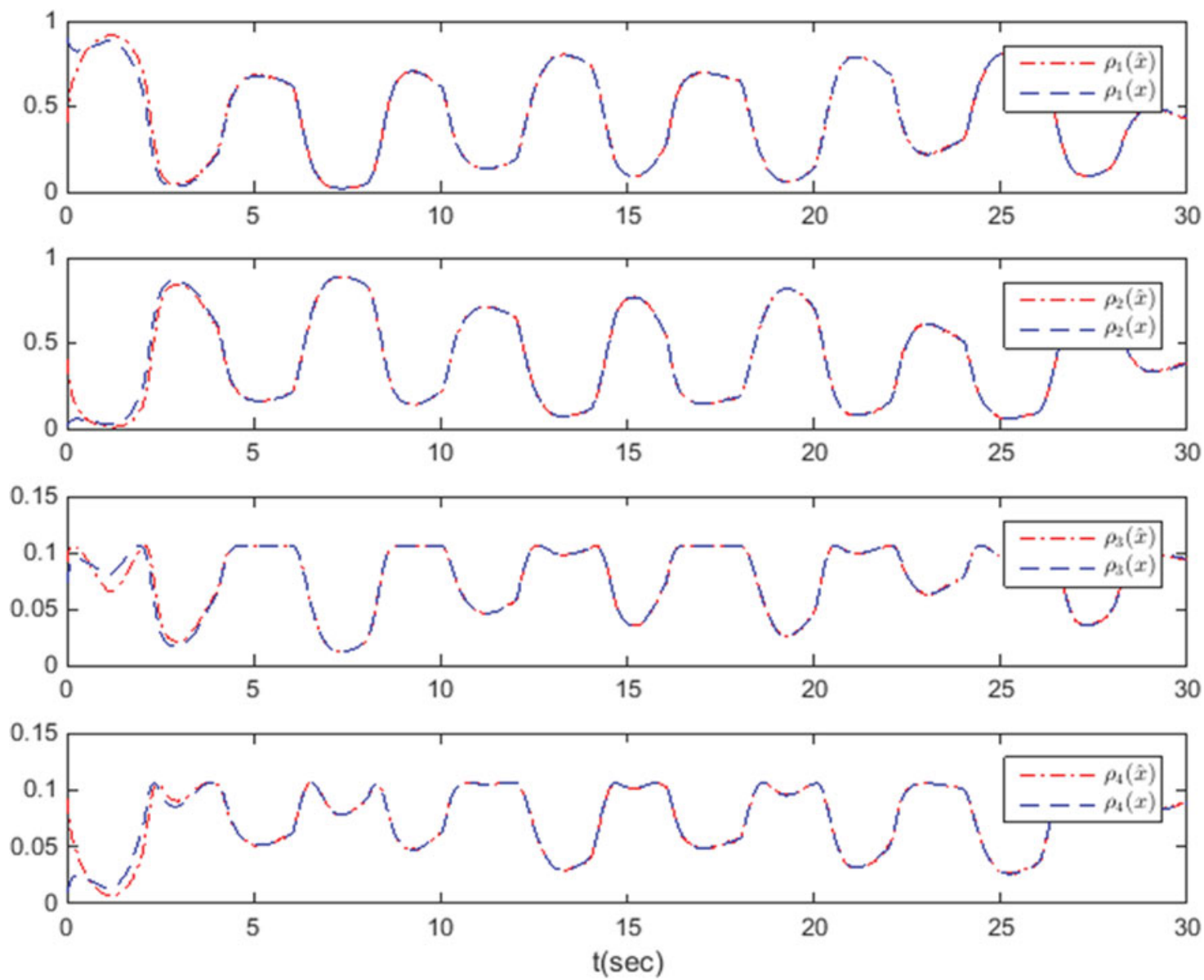

Figure 4. The scheduling functions of the system and the UIO in the second scenario.

$$
L_{11}=\left[\begin{array}{cccc}
0.0000 & 0.0000 & -0.2342 & 0.6709 \\
0.0000 & 0.0000 & -0.4070 & 1.1076 \\
0.0000 & 0.0000 & -0.5411 & 1.8421 \\
0.0000 & 0.0000 & -0.0614 & 0.2342 \\
-0.0000 & -0.0000 & 0.2956 & -0.9052 \\
-0.0000 & -0.0000 & 1.8076 & -6.2290
\end{array}\right]
$$

\subsection{Simulation for the inexact measured parameters case}

The system under consideration and the designed UIO have been simulated. In the simulation, the system is actuated with the input $u(t)=\cos (0.2 t)$. The time variation of the two parameters are $\theta_{1}(t)=\sin (0.3 t)$ and $\theta_{2}(t)=1.5 \cos (0.8 t)$. The parameter measures $\hat{\theta}_{1}(t)$ and $\hat{\theta}_{2}(t)$ are corrupted with two zero-mean noises with standard deviations of 0.2 and 0.3 , respectively. The measurement noise $n(t)$ is considered as a zero-mean noise with standard deviation of 0.1 and the disturbance signal is considered as a zero-mean noise with standard deviation of 0.2 . The vector of faults is $f(t)=$ $\left[f_{1}(t) f_{2}(t)\right]^{T}$. Considering the fault distribution matrix $D_{f}$, faults $f_{1}(t)$ and $f_{2}(t)$ affect the first and the second sensor of the system, respectively. In the first scenario, abrupt fault, incipient fault and sinusoidal fault occur on the two sensors as follows:

$$
\begin{gathered}
f_{1}(t)=\left\{\begin{array}{cc}
1 & 3 \leq t<7 \mathrm{~s} \\
(t-11) / 4 & 11 \leq t<15 \mathrm{~s} \\
\sin (3 t) & 19 \leq t<23 \mathrm{~s} \\
0 & \text { otherwise }
\end{array}\right. \\
f_{2}(t)=\left\{\begin{array}{cc}
1 & 5 \leq t<9 \mathrm{~s} \\
(t-13) / 4 & 13 \leq t<17 \mathrm{~s} \\
\sin (3 t) & 21 \leq t<25 \mathrm{~s} \\
0 & \text { otherwise }
\end{array}\right.
\end{gathered}
$$

The system and the estimated states are depicted in Figure 1. As it can be observed from this figure, the estimated states converge to real states in both fault free and faulty situations despite the presence of noise, disturbance, sensor fault and mismatch between the system scheduling parameters and the UIO scheduling 

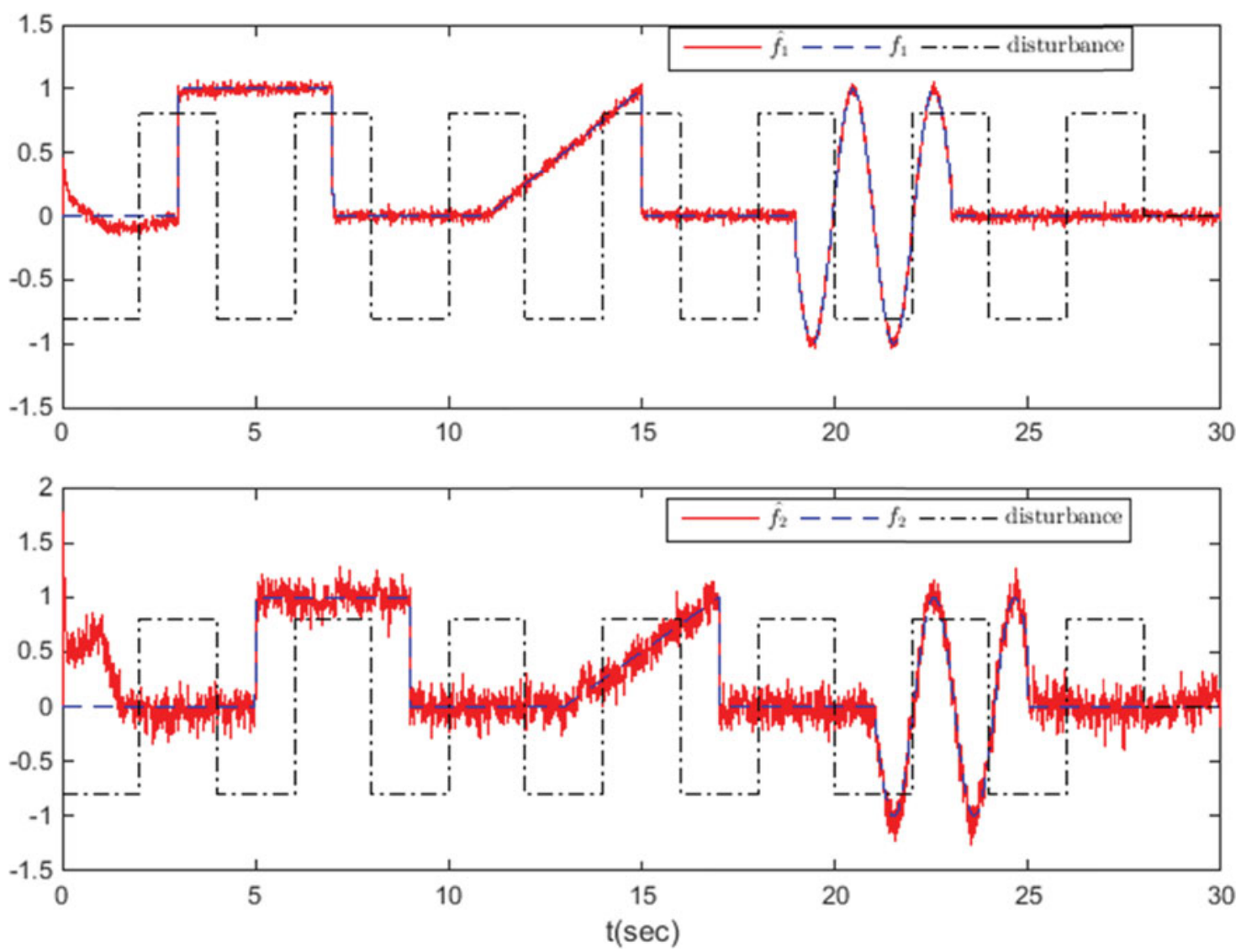

Figure 5. Estimation of the faults on the first and the second sensor in the second scenario.

parameters. In Figure 2, the fault estimates in this scenario are depicted. Note from this plot that the effect of unknown inputs and the noisy parameters are attenuated on the fault estimates. Moreover, the faults on the two sensors are detected and isolated from their estimated values as they occur. In this scenario, in the time intervals between 5-7 seconds, 13-15 seconds and 21-23 seconds, the faults appear simultaneously in the two sensors. Isolation of the simultaneous faults in these intervals has been successfully achieved using the fault estimation approach that is proposed in this paper. Fast detection and isolation of constant and time-varying faults allows to carry out suitable fault tolerant actions manually or automatically to prevent failures in the system.

\subsection{Simulation for the unmeasurable set of parameters case}

In this part, it is assumed that the singular delayed LPV system (5) is scheduled with $\rho_{i}(x(t))$ and according to Remark 4.5, sensor fault diagnosis is performed for this case. The numerical values for different subsystems are similar to the numerical values of Section 5.1 calculated based on (66). The scheduling functions that depend on the unmeasurable variable $x(t)$ are considered as follows:

$$
\rho_{i}(x(t))=\frac{\mu_{i}(x(t))}{\sum_{i=1}^{4} \mu_{i}(x(t))}
$$

for $i=1, \ldots, 4$ where

$$
\begin{gathered}
\mu_{1}(x(t))=\exp \left(2\left(x_{1}(t)+1\right)^{2}\right) \\
\mu_{2}(x(t))=\exp \left(2\left(x_{1}(t)-1\right)^{2}\right) \\
\mu_{3}(x(t))=\exp \left(2\left(x_{1}(t)+0.5\right)^{2}\right) \\
\mu_{4}(x(t))=\exp \left(2\left(x_{1}(t)-0.5\right)^{2}\right) .
\end{gathered}
$$

In this case, the UIO (8) is scheduled based on $\rho_{i}(\hat{x}(t))$. With the UIO that was designed in Section 5.2, a second scenario is simulated. In this scenario, the faults on the two sensors and the measurement noise are considered similar to the first scenario but the disturbance is assumed to be a pulse as it is presented in Figure 5. The real states and the estimated states are shown in Figure 3 where it can be seen that the estimated states converge to real ones with a bounded error. The convergence of the UIO scheduling functions $\rho_{i}(\hat{x}(t))$ to the system scheduling 
functions $\rho_{i}(x(t))$ is shown in Figure 4. Figure 5 depicts the fault diagnosis task in this scenario despite the presence of unknown inputs and uncertainty induced by the unmeasurable parameters in the system. Fault diagnosis is obtained directly via a fault estimation procedure which combines the two phases of detection and isolation in a single phase, avoiding the residual computation and evaluation. Thus, the computation burden of the fault diagnosis unit is remarkably reduced. In addition, it can be observed from the results that perfect decoupling of the unknown input signals $d(t)$ and $f(t)$ on the estimation has been obtained due to constraints (22)-(23) in the design procedure while the measurement noise and the effect of parameters inexactness have been attenuated in the $H_{\infty}$ manner. Perfect decoupling of $f(t)$ allows to isolate the faults that appear simultaneously as shown in Figure 5.

\section{Conclusion}

In this paper, a UIO-based fault diagnosis scheme for singular delayed LPV systems with sensor faults, disturbances, measurement noise and inexact measured parameters has been designed. The sensor faults have been considered as additional states in an augmented system. A UIO was designed for estimation of the new system states in the presence of unknown inputs including disturbances, noise and faults added by the uncertainty which is induced by inexact parameters. The mismatch between real and measured parameters is considered via an uncertain system approach. The robust state estimation for the uncertain system with unknown inputs is formulated using the BRL for the singular delayed LPV system. Fault diagnosis was achieved via fault estimation which is an alternative to methods that require both detection and isolation phases. The results of this paper are also applicable to the singular delayed LPV systems with unmeasurable scheduling functions. Further research might be conducted to extend the results presented in this paper to the multiple delays case and to obtain delay dependent conditions for UIO design and fault diagnosis of singular delayed LPV systems in LMI format by resolving the nonlinearities which appear when using delay dependent robust stability conditions for guaranteeing robust convergence of the proposed UIO. Finally, applying the results obtained in this paper to design an active fault tolerant controller for singular delayed LPV systems will also be considered in the future.

\section{Note}

1. In the following notation, the dimension of matrix blocks is represented as subscripts for the sake of clarity.

\section{Disclosure statement}

No potential conflict of interest was reported by the authors.

\section{Notes on contributors}

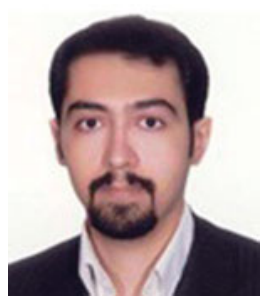

Amir Hossein Hassanabadi received B.Sc. degrees in control systems engineering and electronics respectively in 2007 and 2009 both from Amirkabir University of Technology (Tehran Polytechnic), Tehran, Iran. He received M.Sc. degree and Ph.D. degree in control systems engineering respectively in 2010 and 2016 from Amirkabir University of Technology. He is currently an assistant professor in Electrical Engineering Department of Qazvin Islamic Azad University (QIAU). His current research interests are singular systems, robotics, time delay systems, linear parameter varying (LPV) systems and fault diagnosis.

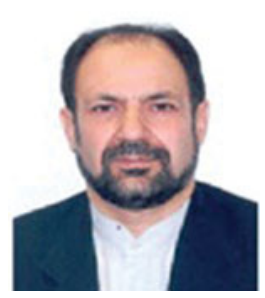

Masoud Shafiee was born in 1957. He Received the BS degree in mathematics, College of Management and Mathematics, Karaj, Iran, 1978, the MS degrees in mathematics (1981) and system engineering (1983) from Write State University, Dayton, Ohio, USA. He also received MS and $\mathrm{PhD}$ degrees in electrical engineering from Louisiana State University, Baton Rouge, Louisiana, USA, in 1985 and 1987, respectively. In 1987 he joined the Department of Electrical Engineering (EE), Amirkabir University of Technology where he is currently a full Professor of EE. His current research interests are in multidimensional (M-D) system, singular systems, robotics and network stability. He is the author/co-author of over 180 research papers and 12 books (as author) and 10 books (as translator) in the areas of signals, systems and mathematics. Professor Shafiee has held numerous positions during his career and established the first Electrical Engineering Conference in Iran in 1993. He has been associated with all Electrical Engineering Conferences in Iran. He was a guest lecturer at different universities including UMIST, University of Sheffield and Manchester Metropolitan University (UK) and also a symposium on "Twodimensional Singular Systems" (UMIST). He is a member of the executive board of Society of Iranian Control and Instrument Engineers, editorial board of Iranian journal of electrical \& electronic engineering, Journal of Control, Iranian Journal of Science and Technology. He is also editor-in-chief of Journal of Information Systems and Telecommunication and Trilateral Congress and editor of seven Conferences and Proceeding of Iranian Electrical Engineering Conference and Trilateral Congress. He is a member of IEEE Control Systems Society Technical Committee on IFAC and ICEE.

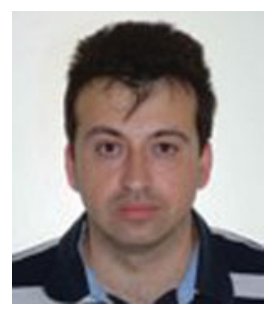

Vicenç Puig received a telecommunications engineering degree in 1993 and a $\mathrm{PhD}$ degree in automatic control, vision, and robotics in 1999, both from Universitat Politècnica de Catalunya (UPC). He is full professor at the Automatic Control Department and a researcher at Institut de Robòtica i Informàtica Industrial at UPC. $\mathrm{He}$ is the chair of the Automatic Control 
Department and the head of the research group on advanced control systems (SAC) at UPC. He has developed important scientific contributions in the areas of fault diagnosis and fault tolerant control using interval and linear-parameter-varying models with set-based approaches. He has participated in more than 20 European and national research projects in the last decade. He has also led many private contracts with several companies and has published more than 100 journal articles as well as 350 papers in international conference/workshop proceedings. $\mathrm{He}$ has supervised over $18 \mathrm{PhD}$ dissertations and more than 40 Master's theses/final projects. He is currently the vice-chair of the IFAC Safeprocess TC Committee 6.4 (2014-2017). He is the chair of the 3rd IEEE Conference on Control and Fault-Tolerant Systems (Systol 2016) and the IPC chair of IFAC Safeprocess 2018.

\section{References}

Ahmadizadeh, S., Zarei, J., \& Karimi, H. R. (2014). Robust unknown input observer design for linear uncertain time delay systems with application to fault detection. Asian Journal of Control, 16(4), 1006-1019.

Aouaouda, S., Chadli, M., Cocquempot, V., \& Tarek Khadir, M. (2013). Multi-objective $\mathrm{H}-/ \mathrm{H} \infty$ fault detection observer design for Takagi-Sugeno fuzzy systems with unmeasurable premise variables: Descriptor approach. International Journal of Adaptive Control and Signal Processing, 27(12), 1031-1047.

Astorga-Zaragoza, C. M., Theilliol, D., Ponsart, J. C., \& Rodrigues, M. (2012). Fault diagnosis for a class of descriptor linear parameter-varying systems. International Journal of Adaptive Control and Signal Processing, 26(3), 208223.

Bokor, J., \& Szabó, Z. (2009). Fault detection and isolation in nonlinear systems. Annual Reviews in Control, 33(2), 113123.

Botmart, T., \& Niamsup, P. (2010). Robust exponential stability and stabilizability of linear parameter dependent systems with delays. Applied Mathematics and Computation, 217(6), 2551-2566.

Chen, J., \& Patton, R. J. (2012). Robust model-based fault diagnosis for dynamic systems. Springer Science \& Business Media, New York.

Chen, L., Zhong, M.-Y., \& Zhang, M.-Y. (2011). Hळ fault detection for linear singular systems with time-varying delay. International Journal of Control, Automation and Systems, 9(1), 9-14.

Dai, L. (1989). Singular control systems. Springer-Verlag, New York.

de Oca, S., Puig, V., Witczak, M., \& Dziekan, L. (2012). Faulttolerant control strategy for actuator faults using LPV techniques: Application to a two degree of freedom helicopter. International Journal of Applied Mathematics and Computer Science, 22(1), 161-171.

De Persis, C., \& Isidori, A. (2001). A geometric approach to nonlinear fault detection and isolation. IEEE Transactions on Automatic Control, 46(6), 853-865.

De Souza, C., Trofino, A., \& De Oliveira, J. (2003). Parametric Lyapunov function approach to $\mathrm{H} 2$ analysis and control of linear parameter-dependent systems. IET Control Theory and Applications, IEE Proceedings, 150(5), pp. 501-508.
Ding, S. X. (2008). Model-based fault diagnosis techniques: Design schemes, algorithms, and tools. Springer Science \& Business Media, Berlin.

Duan, G.-R., Howe, D., \& Patton, R. J. (2002). Robust fault detection in descriptor linear systems via generalized unknown input observers. International Journal of Systems Science, 33(5), 369-377.

Estrada, F. L., Ponsart, J., Theilliol, D., \& Astorga-Zaragoza, C. (2015). Robust $\mathrm{H}-/ \mathrm{H} \infty$ fault detection observer design for descriptor-LPV systems with unmeasurable gain scheduling functions. International Journal of Control, 88(11), 2380-2391.

Frank, P. M. (1994). On-line fault detection in uncertain nonlinear systems using diagnostic observers: A survey. International journal of systems science, 25(12), 2129-2154.

Gao, Z., \& Ding, S. X. (2007). Actuator fault robust estimation and fault-tolerant control for a class of nonlinear descriptor systems. Automatica, 43(5), 912-920.

Giarré, L., Bauso, D., Falugi, P., \& Bamieh, B. (2006). LPV model identification for gain scheduling control: An application to rotating stall and surge control problem. Control Engineering Practice, 14(4), 351-361.

Hamdi, H., Rodrigues, M., Mechmeche, C., \& Braiek, N. B. (2012a). Robust fault detection and estimation for descriptor systems based on multi-models concept. International Journal of Control, Automation and Systems, 10(6), 12601266.

Hamdi, H., Rodrigues, M., Mechmeche, C., Theilliol, D., \& Braiek, N. B. (2012b). Fault detection and isolation in linear parameter-varying descriptor systems via proportional integral observer. International journal of adaptive control and signal processing, 26(3), 224-240.

Hassanabadi, A. H., Shafiee, M., \& Puig, V. (2016a). Robust fault detection of singular LPV systems with multiple timevaring delays. International Journal of Applied Mathematics and Computer Science, 26(1), 45-61.

Hassanabadi, A. H., Shafiee, M., \& Puig, V. (2016b). UIO design for singular delayed LPV systems with application to actuator fault detection and isolation. International Journal of Systems Science, 47(1), 107-121.

Ichalal, D., Marx, B., Ragot, J., \& Maquin, D. (2009). Fault diagnosis in Takagi-Sugeno nonlinear systems. IFAC Proceedings Volumes 42(8), pp. 504-509.

Jetto, L., \& Orsini, V. (2010). Efficient LMI-based quadratic stabilization of interval LPV systems with noisy parameter measures. IEEE Transactions on Automatic Control, 55(4), 993-998.

Jiang, B., \& Chowdhury, F. N. (2005). Parameter fault detection and estimation of a class of nonlinear systems using observers. Journal of the Franklin Institute, 342(7), 725-736.

Karimi, H. R. (2006). Robust stabilization with $\mathrm{H} \infty$ performance for a class of linear parameter-dependent systems. Mathematical Problems in Engineering, 2006, 15.

Koenig, D. (2005). Unknown input proportional multipleintegral observer design for linear descriptor systems: Application to state and fault estimation. IEEE Transactions on Automatic Control, 50(2), 212-217.

Lam, J., \& Xu, S. (2006). Robust control and filtering of singular systems. Berlin/Heidelberg: Springer-Verlag.

Li, F., \& Zhang, X. (2012). A delay-dependent bounded real lemma for singular LPV systems with time-variant delay. International Journal of Robust and Nonlinear Control, 22(5), 559-574. 
Li, F., \& Zhang, X. (2013). Delay-range-dependent robust $\mathrm{H} \infty$ filtering for singular LPV systems with time variant delay. International Journal of Innovative Computing, Information and Control, 9(1), 339-353.

Löfberg, J. (2004). YALMIP: A toolbox for modeling and optimization in MATLAB. Taipei: IEEE International Symposium on Computer Aided Control Systems Design, IEEE.

López-Estrada, F.-R., Ponsart, J.-C., Astorga-Zaragoza, C.-M., Camas-Anzueto, J.-L., \& Theilliol, D. (2015). Robust sensor fault estimation for descriptor-LPV systems with unmeasurable gain scheduling functions: Application to an anaerobic bioreactor. International Journal of Applied Mathematics and Computer Science, 25(2), 233-244.

López-Estrada, F. R., Ponsart, J.-C., Astorga Zaragoza, C. M., Theilliol, D., \& Aberkane, S. (2014). Fault diagnosis based on robust observer for Descriptor-LPV Systems with unmeasurable scheduling functions. Cape Town: 19th IFAC World Congress.

Masubuchi, I., Kato, J., Saeki, M., \& Ohara, A. (2004). Gainscheduled controller design based on descriptor representation of LPV systems: Application to flight vehicle control. 43rd IEEE Conference on Decision and Control, IEEE, Nassau, Bahamas.

Meskin, N., \& Khorasani, K. (2009a). Fault detection and isolation of distributed time-delay systems. IEEE Transactions on Automatic Control, 54(11), 2680-2685.

Meskin, N., \& Khorasani, K. (2009b). Robust fault detection and isolation of time-delay systems using a geometric approach. Automatica, 45(6), 1567-1573.

Moodi, H., \& Farrokhi, M. (2013). Robust observer-based controller design for TS systems with nonlinear consequent parts. Iran: 21st Iranian Conference on Electrical Engineering (ICEE), Mashhad, Iran: IEEE.

Rodrigues, M., Hamdi, H., Braiek, N. B., \& Theilliol, D. (2014). Observer-based fault tolerant control design for a class of
LPV descriptor systems. Journal of the Franklin Institute, 351(6), 3104-3125.

Rodrigues, M., Sahnoun, M., Theilliol, D., \& Ponsart, J.-C. (2013). Sensor fault detection and isolation filter for polytopic LPV systems: A winding machine application. Journal of Process Control, 23(6), 805-816.

Shamma, J. S. (1988). Analysis and design of gain scheduled control systems (PhD thesis). Massachusetts Institute of Technology, Cambridge, MA.

Sturm, J. F. (1999). Using SeDuMi 1.02, a MATLAB toolbox for optimization over symmetric cones. Optimization methods and software, 11(1-4), 625-653.

Theilliol, D., \& Aberkane, S. (2011). Desing of LPV observers with unmeasurable gain scheduling variable under sensors faults. Milano: IFAC World Congress.

Wang, Z., Rodrigues, M., Theilliol, D., \& Shen, Y. (2015). Actuator fault estimation observer design for discrete-time linear parameter-varying descriptor systems. International Journal of Adaptive Control and Signal Processing, 29(2), 242258.

Yang, F., Wang, Z., Hung, Y., \& Shu, H. (2005). Mixed H 2/Ho filtering for uncertain systems with regional pole assignment. IEEE Transactions on Aerospace and Electronic Systems, 41(2), 438-448.

Yoneyama, J. (2009). Hळ filtering for fuzzy systems with immeasurable premise variables: An uncertain system approach. Fuzzy Sets and Systems, 160(12), 1738-1748.

Zhai, D., Zhang, Q.-L., \& Li, J.-H. (2014). Fault detection for singular multiple time-delay systems with application to electrical circuit. Journal of the Franklin Institute, 351(12), 5411-5436.

Zhang, X., \& Zhu, H. (2012). Robust stability and stabilization criteria for discrete singular time-delay LPV systems. Asian Journal of Control, 14(4), 1084-1094. 